\title{
Mobilität als ÜBerLebensSTRATEgie: Die lange Fluchtmigration der Afghan/Inn/en
}

\author{
Gabriele Rasuly-PaLECzeK, Wien, Institut für Kultur- und Sozialanthro- \\ pologie, Universität Wien
}

\section{INHALT}

1 Einleitung

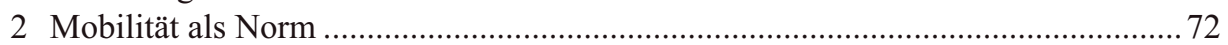

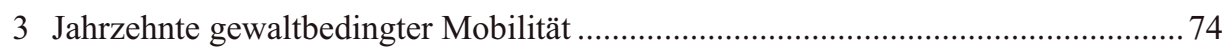

3.1 Erste Fluchtmigrationsphase: vom kommunistischen Putsch bis zum Sieg der Mujaheddin: (1978/1979-1989/1992).................................. 74

3.2 Zweite Fluchtmigrationsphase:

Während der Mujaheddin-Herrschaft (1992-1996) .........................................75

3.3 Dritte Fluchtmigrationsphase: Während der Taliban-Ära (1996-2001)..............76

3.4 Vierte Fluchtmigrationsphase: Post-Taliban-Ära

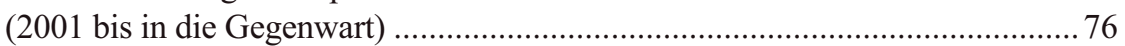

4 Faktoren der rezenten Mobilität ............................................................................. 81

5 Multiple Wanderungen/Migrationen als Überlebensstrategie ....................................86

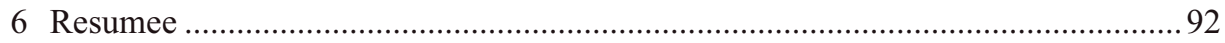

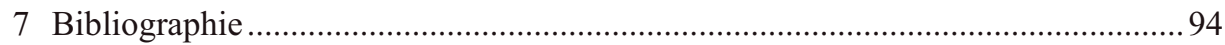

\section{Einleitung}

Laut Angaben des UNHCR befinden sich der derzeit weltweit rund 68,5 Mill. Menschen auf der Flucht ${ }^{1}$. Davon gelten rund 13,4 Mill. als sogenannte „Protracted 1 In den Statistiken des UNHCR werden nur jene Personen berücksichtigt, die vor Verfolgung, Konflikten oder allgemeiner Gewalt fliehen (UNHCR 2018a, p. 2). Nicht erfasst werden darin jene Personen, die infolge von Naturkatastrophen wie Dürre, Erdbeben, Stürmen etc. fliehen. 2016 waren dies laut Angaben des IDMC (2017: p. 32) rund 23,5 Mill. 
Refugees", d.h. als Personen, die seit mindestens 5 Jahren in einem Asylland leben. ${ }^{2} \mathrm{Zu}$ ihnen zählen Millionen von Afghan/inn/en, die nach Flüchtlingen aus Syrien (6,3 Mill.) die zweitgrößte Flüchtlingsgruppe (2,6 Mill.) darstellen (UNHCR 2018 a). ${ }^{3}$

Seit fast vier Jahrzehnten prägen Displacement, Flucht, Exil, Rückkehr und erneute Flucht den Alltag eines großen Teils der afghanischen Gesellschaft. Für unzählige Afghan/inn/en sind seither unterschiedliche Formen der Mobilität zu einer essentiellen Überlebensstrategie geworden (vgl. BERG HARPVIKEN 2014; HAKIMI 2016; Monsutti 2008, 2018b; Schmeidl 2019). ${ }^{4}$ Diese inkludieren nicht nur diverse Fluchtbewegungen wegen persönlich erlittener Bedrohung und eskalierender Gewalt (z.B. kurzfristige Fluchtmigration innerhalb Afghanistans oder permanente Migration ins Ausland), sondern umfassen auch andere Migrationsformen, wie z.B. saisonale oder permanente Arbeitsmigration als Folge kriegsbedingter Verarmung oder sich verschlechternder Lebensbedingungen am gegenwärtigen Residenzort, Weiterwanderung in ein anderes Asylland wegen der immer restriktiver werdenden Flüchtlingspolitik im aktuellen Aufnahmeland (z.B. Migration aus dem Iran in ein europäisches Land) oder auch nur eine kurzfristige Rückkehr nach Afghanistan (z.B. in den 1980er Jahren saisonale Migration der Männer aus pakistanischen Flüchtlingslagern, um am Widerstandskampf in Afghanistan teilzunehmen, oder heute Kurzbesuche in Afghanistan, um Ressourcen im Herkunftsgebiet zu akquirieren, z.B. Einnahmen aus Verpachtungen von Grundstücken oder Häusern).

Die vielfältigen Migrationsformen und Mobilitätsstrategien derer sich Afghan/ inn/en seit Beginn der kriegerischen Konflikte (1978/79) bedienen, sind ein gutes Beispiel dafür, dass die bislang sowohl im wissenschaftlichen Diskurs wie auch in der politischen Debatte häufig vorgenommene Differenzierung zwischen Flucht und Migration bzw. „forced migration“ und „voluntary migration“ oder zwischen Flüchtlingen im Sinne der Genfer Konvention und sogenannten Wirtschaftsflüchtlingen bzw. „genuine refugees" und „bogus refugees" kaum zielführend und daher kritisch zu reflektieren ist (vgl. Monsutti 2008, 2018a, pp. 450, 454 und 2018b; Grawert und Mielke 2018, pp. 9, 38; Fiddian-Qasmiyen et al. 2014, p. 4 f.; Aleinikoff 2017). ${ }^{5}$

„UNHCR defines a protracted refugee situation as one in which 25.000 or more refugees from the same nationality have been in exile for five consecutive years or more in a given asylum country." (UNHCR 2018a, p. 22); vgl. auch Grawert und MielKe 2018, p. 7.

Neben den 2,6 Mill. offiziell registrierten afghanischen Flüchtlingen leben ca. 1,5 bis 2 Mill. nicht-dokumentierter Flüchtlinge im Iran und zwischen 350.000 und 550.000 in Pakistan (UNHCR 2018b, p. 4; IOM und UNHCR 2018, p. 4).

Das Leben vieler Afghan/inn/en war immer stark von Mobilität geprägt (vgl. MonsutTi 2008; SCHMEIDL 2019; SCHETTER 2012; MARSDEN 2016; Details siehe unten).

Ad. Differenzierung in „,bogus” und „genuine refugees“ vgl. Diop 2014; Neumayer 2005; Betts 2013a, 2013b; BaKewell 2011; Castles 2013; Kleist 2015a, 2018a; Koser und Martin 2011; Zettler 2007. Ad. Diskurs in der österr. Politik vgl. Scheibelhofer 2017, v.a. p. 104 f.; Matzenberger und Minkin 2016; Treichelt und Gepp 2015. 
Die sehr eng gefasste Definition des Begriffs Flüchtling der Genfer Flüchtlingskonvention wird der großen Bandbreite rezenter Fluchtursachen (z.B. Displacement infolge des Klimawandels, sekundäre Migration wegen der Unmöglichkeit nach der Rückkehr aus dem Exil im Herkunftsland wieder Fuß zu fassen) nicht gerecht. ${ }^{6}$

Zahlreiche Migrations- und Fluchtforscher/innen gehen heute von multiplen Migrations- bzw. Fluchtursachen aus (FIDDIAN-QASMIYeH et al. 2014; BRÜCKER et al. 2016; Crawley et al. 2016; Donini et al. 2016; Betts 2013a, pp. 16-18; Castles 2013; KLEIST 2015a, 2018a; ZetTer 2007) und plädieren für eine Abkehr von Kategorisierungsversuchen (vgl. Monsutti 2008; Grawert und Mielke 2018; Aleinikoff 2017).

Am Beispiel der seit 1978/79 vonstattengehenden Migrationsbewegungen der Afghan/inn/en soll im Folgenden die Bedeutung der Mobilität als Überlebensstrategie illustriert werden und damit ein Beitrag zur Debatte um die Sinnhaftigkeit von Differenzierungen zwischen „,forced“ und ,voluntary migration“ bzw. „bogus“ und „,genuine refugees" geleistet werden.

Nach einer kurzen Darstellung der allgemeinen Bedeutung von Mobilität in der afghanischen Gesellschaft und einem Exkurs über den Umfang der seit fast vier Jahrzehnten andauernden konfliktbedingten Migrationsbewegungen wird v.a. auf jene Faktoren verwiesen werden, die eine Mobilitätsentscheidung beeinflussen. Des Weiteren sollen die unterschiedlichen Mobilitätsstrategien (z.B. kurzfristige Flucht, saisonale Arbeitsmigration im Asylland, sekundäre Migration etc.) unter dem Gesichtspunkt der Überlebenssicherung dargelegt werden. Dabei wird sowohl auf die vorhandene Sekundärliteratur zur rezenten Mobilität von Afghan/inn/en sowie auf mehrere seit 2015 gemeinsam mit anderen Wiener Kolleg/inn/en durchführte empirische Erhebungen unter afghanischen Asylwerber/inne/n und anerkannten Flüchtlingen Bezug genommen werden. ${ }^{7}$

6 Ad. Kategorisierung unterschiedlicher Flüchtlingsgruppen vgl. IOM 2016; IDMC 2017; Migration Data Portal 2019a, 2019b. Das IOM nimmt in seinem „Baseline Mobility Assessment" ebenfalls eine grundlegende Differenzierung unterschiedlicher Gruppen von Afghan/inn/ en vor und unterscheidet z.B. zwischen „Returnees from Abroad, Out-Migrants, Arrival IDP's, Returned IDP's and Fled IDP's“ (IOM 2019b). Ad. Diskurs um unterschiedliche Gruppen von Flüchtlingen vgl. Betts 2013a, 2013b; Chatty und Marfleet 2013; Kleist 2015a, 2018b; Turton 2003a, 2003b; ZeTTLER 2007.

Insgesamt wurden bislang drei Studien durchführt. Zum einen eine großteils vom ISR finanzierte Pilotstudie in der zwischen Dezember 2015 und April 2016 insgesamt 20 Afghan/inn/en unterschiedlichen ethno-linguistischen und religiösen Backgrounds befragt wurden (Details siehe Kohlbacher und Rasuly-Paleczek 2018 und Rasuly-Paleczek 2017). Zum anderen eine vom BMEIA finanzierte Befragung von 65 Afghan/inn/en unterschiedlicher Aufenthaltsdauer in Österreich sowie von 13 Expert/inn/en, die zwischen April 2017 und März 2018 in Wien, Nieder- und Oberösterreich durchgeführt wurde (Details siehe FrIEDRICH et al. 2019) und schließlich das seitens der ÖAW finanzierte „LODA-Projekt“, in dessen Verlauf 33 Afghan/inn/en befragt wurden. 


\section{Mobilität als Norm}

Flucht, Vertreibung und Arbeitsmigration stellen keine neuen Phänomene in der Geschichte Afghanistans dar. Schon vor dem Einsetzen der kriegsbedingten Migrationsbewegungen vor vier Jahrzehnten waren große Teile der afghanischen Bevölkerung äußerst mobil, sei es als Nomad/inn/en, saisonale Arbeitskräfte, Pilger/innen, Student/ inn/en und Händler oder als Personen, die vor Dürre und anderen Naturkatastrophen ${ }^{8}$ oder lokalen politischen Auseinandersetzungen in andere Landesteile oder ins Ausland flohen (vgl. Centlivres und Centlivres-Demont 1988; Schetter 2012; Monsutti 2005, 2008 und 2018 b).

Insbesondere der Arbeitsmigration innerhalb Afghanistans wie auch ins Ausland kam eine zunehmend größere Bedeutung zu. Bereits im 19. Jahrhundert hatten einige Hazara wegen der prekären Wirtschaftslage in ihrem zentralafghanischen Siedlungsgebiet - dem Hazarajat - begonnen saisonal nach Kabul zu migrieren, um als Lastenträger, Hausgehilfen etc. ein zusätzliches Einkommen zu erwirtschaften. ${ }^{9}$ Im Verlauf des 20. Jahrhunderts zwang die zunehmende Verschlechterung ihrer Lebensbedingungen immer mehr Hazara dazu ihre Heimatregion zu verlassen und in den Städten Afghanistans oder im Ausland, vor allem im Gebiet von Quetta und Meschad, wo seit der Verfolgungspolitik Amir Abdurrahman Khans eine wachsende Hazara-Diaspora entstanden war, nach Arbeit zu suchen (vgl. Monsutti 2005; IBRAHim 2017).

Auch in anderen Landesteilen kam es zu einem deutlichen Anstieg der Arbeitsmigration, so z.B. in den Baumwollanbaugebieten um Kunduz/NO-Afghanistan, deren erhöhter Arbeitskräftebedarf zahlreiche saisonale und permanente Arbeitsmigranten (z.B. aus den Hochgebirgsregionen des Hindukusch) anzog (GrötzBACH 1972; CENTLivres und Centlivres-Demont 1988, pp. 65-77). Ab den frühen 1970er Jahren gewann zudem die Arbeitsmigration ins Ausland stetig an Bedeutung. Zahlreiche Afghanen (neben den Hazara nun auch Angehörige anderer ethnischer Gruppen, z.B. Paschtunen und Tadschiken) begannen auf der Suche nach Arbeit in die beiden Nachbarstaaten Iran und Pakistan sowie in einige arabische Golfstaaten zu migrieren (vgl. SCHETTER 2012; SCHMEIDL 2019).

Neben der Arbeitsmigration erlangte ab Mitte des 20. Jahrhunderts außerdem die Migration zu Studienzwecken eine zunehmend größere Relevanz. Dank internationaler

\footnotetext{
$8 \quad$ Lang andauernde Dürreperioden (z.B. 1970-1972, 1998-2001, 2008-2011, 2018) führen immer wieder zu internen Wanderbewegungen. Beispielsweise lebten um 1998 allein im Gebiet der Stadt Herat zwischen 80.000 bis 100.000 Personen in IDP-Camps. Sie waren wegen der Dürre aus ihren besonders betroffenen Nachbarprovinzen zugezogen (vgl. BRAAKMAN 2005, p. 18).

Die afghanische Gesellschaft ist durch eine erhebliche soziokulturelle, sprachliche und religiöse Vielfalt geprägt. Zu den zahlenmäßig größten Gruppen gehören die Paschtunen, Tadschiken, Hazara und Uzbeken, vgl. Orywal 1986 und Vogelsang 2002. Im vorliegenden Text sind unter den jeweiligen Gruppenbezeichnungen sowohl Frauen wie auch Männer inkludiert.
} 
Entwicklungshilfe konnten zahlreiche junge Afghan/inn/en in Großbritannien, Frankreich, Deutschland, den USA und der Sowjetunion ein Studium absolvieren. Sie bildeten einen wesentlichen Teil der seit den 1960er Jahren stetig wachsenden modern gebildeten Ober- und Mittelschicht (RAsuly 1997).

Mobilität wurde seitens der politisch Mächtigen wiederholt auch erzwungen und zur Herrschaftsausübung instrumentalisiert, indem aufständische Bevölkerungsgruppen nach der Niederschlagung ihrer Rebellionen gegen die afghanische Zentralregierung in andere Landesteile deportiert oder zur Flucht in die Nachbarländer gezwungen und missliebige Persönlichkeiten ins Ausland exiliert wurden. ${ }^{10}$ Beispielsweise ließ Amir Abdurrahman Khan (1880-1901) im Zuge seiner Zentralisierungsbemühungen die Ghilzai-Paschtunen, Hazara und Uzbeken in andere Landesteile deportieren oder zwang sie (z.B. die Hazara) zur Flucht ins Ausland. ${ }^{11}$

Migrationsbewegungen sind somit - wie MonsutTi (2008, p. 60) konstatiert „part of the Afghan social and cultural landscape" und stellten bzw. stellen eine wesentliche Strategie der Überlebenssicherung vieler Afghan/inn/en dar.

Mit den durch den Putsch der VDPA im April 1978 und der sowjetischen Invasion im Dezember 1979 ausgelösten und bis heute andauernden gewaltsamen Auseinandersetzungen erreichte die Mobilität der Afghan/inn/en schließlich eine Dimension, welche die afghanische Gesellschaft nachhaltig geprägt und verändert hat. Für Millionen Afghan/inn/en sind Flucht, Exil und Rückkehr - bisweilen mehrfach - seither zu einem fixen Bestandteil ihrer Alltagserfahrung und zu einer wichtigen Überlebensstrategie geworden (Berg Harpviken 2014; Hakimi 2016; Schetter 2012; Monsutti 2018b; SchmeIdL 2019). Bis zum Beginn des syrischen Bürgerkriegs stellten Afghan/inn/en 34 Jahre lang die weltweit größte individuelle Flüchtlingsgruppe dar.

Um das Ausmaß dieser seit vier Jahrzehnten andauernden gewaltbedingten Mobilität zu veranschaulichen, soll diese im Folgenden kurz skizziert werden.

10 So wurden Angehörige der herrschenden Dynastie im Verlauf des 19. Jahrhunderts nach Britisch-Indien exiliert oder hochrangige Mitglieder der Volksdemokratischen Partei Afghanistans (VDPA), die im Zuge von parteiinternen Auseinandersetzungen (1978/1979) in Ungnade gefallen waren, auf Botschafterposten ins Ausland abgeschoben (RASUly 1997; BRAAKMAN 2005 und NABY 2005).

11 Allgemein ad. Deportationen vgl. GrötZBach (1990, p. 10 und 1972), ad. Hazara (Monsutti 2005 und Ibrahimi 2017), ad. Uzbeken (Rasuly-PALECZEK 2004), ad. Paschtunen vgl. TAPPer (1983). 


\section{Jahrzehnte gewaltbedingter Mobilität}

Insgesamt können vier große Fluchtmigrationsphasen (1. vom kommunistischen Putsch bis zum Sieg der Mujaheddin (1978-1992), 2. während der Mujaheddin-Herrschaft (1992-1996), 3. während der Taliban-Ära (1996-2001) und 4. Post-Taliban-Ära) unterschieden werden, wobei einzelne zudem Rückkehrbewegungen von Flüchtlingen in die Heimat inkludieren.

Parallel dazu fanden bzw. finden auch andere Formen der Mobilität statt, wie z.B. Binnenmigration wegen Dürre und anderen Naturkatastrophen, periodische Rückkehr der Männer nach Afghanistan, um am Widerstandskampf teilzunehmen, sowie Arbeitsmigration innerhalb des Asyllandes oder ins Ausland (z.B. von Pakistan in einen der arabischen Golfstaaten), um die Lebensbedingungen der in den Flüchtlingslagern zurückgebliebenen Familienangehörigen zu verbessern (GRAWERT und MielKe 2018).

Was den zahlenmäßigen Umfang der einzelnen Fluchtbewegungen angeht, ist darauf hinzuweisen, dass dazu keine exakten Zahlenangaben vorliegen. Sowohl was die Vergangenheit wie auch was die Gegenwart angeht, weisen die Angaben verschiedener staatlicher Behörden (z.B. Afghanistans, des Irans oder Pakistans, aber auch europäischer Staaten) und internationaler Organisationen (z.B. UNHCR, IOM etc.) beträchtliche Schwankungen auf (vgl. HAKIMI 2016, p. 15 f.). ${ }^{12}$ Besonders ungenau sind die Angaben zu den IDP's sowie zu Rückkehrer/inne/n aus dem Iran und Pakistan. ${ }^{13}$

\subsection{Erste Fluchtmigrationsphase: vom kommunistischen Putsch bis zum Sieg der Mujaheddin: (1978/1979-1989/1992)}

Bereits kurz nach dem Putsch der kommunistischen Partei (VDPA) im April 1978 setzte eine erste Auswanderung ein. Ihr gehörten vor allem höhere Beamte früherer Regierungen, islamische Geistliche, reiche Geschäftsleute sowie liberale und maoistische Intellektuelle an, die in Opposition zum Regime standen und von diesem verfolgt wurden, daneben aber auch Personen aus dem Umkreis der VDPA, die im Zuge von parteiinternen Fraktionskämpfen in Ungnade gefallen waren (BRAAKMAN 2005, p. 15; NABY 2005, p. 177; RASUly 1997).

Die meisten von ihnen flohen in der Folge in westliche Länder (BELLER 2013, p. 23). Damit begann ein Trend, der sich in den nächsten Jahrzehnten fortsetzte und dazu

12 Beispielsweise nennt GrötZBACH (1990, p. 60) für Ende 1989 5,5 Mill. Afghan/inn/en, die ins Ausland geflohen sind. HAKIMI (2016, p. 8), der sich auf Zahlen des UNHCR bezieht, gibt ihre Zahl mit 6,2 Mill. an (vgl. auch Beller 2013, p. 25). Selbst die Asylwerber/innen-Statistiken europäischer Staaten weisen erhebliche Lücken und Inkonsistenzen auf (vgl. KLEIST 2015b).

13 Ad. Problem der IDP-Zahlen siehe OCHA 2017b, Glatz 2015, BJelica 2016c; IDMC 2017. Ad. Problem mit Rückkehrer/innenzahlen vgl. Kronenfeld 2008; Turton und Marsden 2002, p. 21 sowie AMNesty InTERNATIONAL 2017, p. 30. 
führte, dass die westlich gebildete Schicht des Landes auf einen Bruchteil ihrer vorrevolutionären Zahl minimiert wurde (BeLLER 2013, p. 23).

Schon bald begannen auch Bauern und Nomaden wegen der in den ländlichen Regionen eskalierenden Gewalt ins benachbarte Ausland (v.a. nach Iran und Pakistan) zu fliehen. Mit der Invasion sowjetischer Truppen im Dezember 1979 kam es zu einem dramatischen Anstieg der Flüchtlingszahlen, von rund 400.000 (Ende 1979) auf ca. 3,9 Mill. im Jahr 1983 (BelLer 2013, p. 24). In den folgenden Jahren nahm ihre Zahl wegen der systematischen Zerstörung von Dörfern, Feldern und Bewässerungsanlagen weiter zu.

Insgesamt sollen offiziellen Angaben zufolge bis Ende 1989 rund 5,5 Mill. bis 6,2 Mill. Afghan/inn/en ins Ausland geflohen sein, die meisten davon in die beiden Nachbarstaaten Pakistan (ca. 3,5 Mill.) und Iran (ca. 2 Mill.) sowie einige hunderttausend auch nach Indien (GrötZBACH 1990, p. 60; НАкIMI 2016, p. 15). Nur ein kleiner Bruchteil kam damals in westliche Länder (in Europa v.a. nach Deutschland, in die USA und Kanada) (Beller 2013; BraAkman 2005).

Basierend auf einer geschätzten Einwohnerzahl von damals ca. 15 Mill. waren demnach 33\% der afghanischen Gesamtbevölkerung ins Ausland geflohen, weitere 11\% zu Binnenflüchtlingen geworden und rund $9 \%$ getötet wurden (SLIwINSKI 1988 zit. nach GrötZBACH 1990, p. 57).

\subsection{Zweite Fluchtmigrationsphase: Während der Mujaheddin- Herrschaft (1992-1996)}

Für die Herrschaftsperiode der Muhajeddin (1992-1996) lassen sich zwei gegenläufige Trends feststellen. Zum einen begann ein Teil der ins benachbarte Ausland geflohenen Afghan/inn/en nach dem Sturz des kommunistischen Regimes im April 1992 in die Heimat zurückzukehren. Laut Angaben des UNHCR sollen 1992 ca. 1,6 Mill. und 1993 rund 1 Mill. zurückgekehrt sein, davon allein aus Pakistan 1,8 Mill. (НАкімі 2016, p. 6).

Zum anderen kam es schon bald nach dem Sieg der Mujaheddin zu gewaltsam ausgetragenen Konflikten zwischen den verschiedenen Mujaheddin-Fraktionen. Insbesondere die Zivilbevölkerung Kabuls war umfassenden Menschenrechtsvergehen (z.B. Entführungen, Massenvergewaltigungen, willkürliche Tötungen) und Bombardierungen ihrer Wohnviertel durch einander bekriegende Mujaheddin-Gruppierungen ausgesetzt. Dieser mit aller Härte ausgetragene Kampf um die Kontrolle der Stadt führte nicht nur zur fast völligen Zerstörung Kabuls und des Zusammenbruches ihres Wirtschaftslebens, sondern auch zu einem umfassenden Exodus der Bewohner/innen, deren Zahl von ca. 3 Mill. auf unter 1 Mill. sank. (Rasuly 1997, p. 9).

Bevorzugtes Zufluchtsgebiet war der Norden Afghanistans, insbesondere die Stadt Mazar-e-Scharif, die bis zu ihrer Einnahme durch die Taliban im Jahr 1998 von mili- 
tärischen Auseinandersetzungen verschont blieb. ${ }^{14}$ Andere Zufluchtsorte waren Jalalabad im Süden und Herat im Westen. Insgesamt sollen Mitte der 1990er Jahre mehr als 400.000 intern Vertriebene in oft nur schlecht versorgten Flüchtlingslagern in der Nähe von Jalalabad, Mazar-e-Scharif und Herat gelebt haben (BJELICA 2016c). Funktionsträger/inn/en und Sympathisant/inn/en des ehemaligen VDPA-Regimes versuchten großteils in den Westen oder nach Russland zu gelangen (vgl. BRAAKMAN 2005, p. 15 f.; BELLER 2013, p. 26 ff.).

\subsection{Dritte Fluchtmigrationsphase: Während der Taliban-Ära (1996-2001)}

Während der Herrschaft der Taliban dauerte die Fluchtmigration an. Ihre repressiven Maßnahmen (z.B. Bildungs- und Berufsverbot für Frauen) und ihre gewaltsamen Übergriffe auf die Zivilbevölkerung, vor allem in Zentral- und Nordafghanistan, wo es 1998 in Mazar-e-Scharif zu einem Massaker an tausenden Hazara und hunderten Uzbek/inn/en kam, setzten neue Fluchtbewegungen in Gang.

Schätzungen zufolge sollen rund 1 Mill. Afghan/inn/en vor den Taliban geflohen sein (vgl. BJelicA 2016c). Die meisten von ihnen (rund 800.000 Personen) suchten damals Schutz im Nordosten des Landes, jenen 10\% Afghanistans, die noch von den Anti-Taliban-Kräften der Nordallianz unter Führung von Ahmad Schah Massud kontrolliert wurden (vgl. Beller 2013, p. 29; Rasuly-Paleczek 2001).

Unter jenen, die im benachbarten Ausland (Iran ca. 100.000 und Pakistan ca. 200.000; BelLeR 2013, p. 29) Schutz suchten, waren - wie in der Vergangenheit - vor allem Personen ländlicher Herkunft sowie Angehörige der städtischen Unterschicht. Afghan/inn/en, die zur gebildeten städtischen Mittel- und Oberschicht zählten, migrierten - wie schon früher - bevorzugt in westliche Länder (Beller 2013; BRAAKMAN 2005).

Eine besonders große Flüchtlingsgruppe waren die schiitischen Muslim/inn/e/n (insbesondere die Hazara und Ismailis) (Centlivres und Centlivres-Demont 2000, p. 157; GeHRIG 1999, p. 184), die in den Augen der radikal sunnitischen Taliban als Abtrünnige vom wahren Islam galten und daher verfolgt wurden, sowie Angehörige der nichtmuslimischen Minderheiten des Landes (v.a. Sikhs und Hindus). Letztere suchten v.a. in Indien Schutz (BeLler 2013, p. 29; BraAKMAN 2005, p. 17).

\subsection{Vierte Fluchtmigrationsphase: Post-Taliban-Ära (2001 bis in die Gegenwart)}

Mit dem Ende des Taliban-Regimes (November 2001 Einnahme Kabuls durch die Nord-Allianz und die mit ihr verbündeten ausländischen Truppen) begannen Millionen

\footnotetext{
14 Wegen der Verschärfung der Flüchtlingspolitik im Iran und in Pakistan wurde die Flucht in diese beiden Staaten zunehmend schwieriger (vgl. RAsuly-PALECZEK 2001).
} 
afghanischer Flüchtlinge - oft nach jahrzehntelangem Aufenthalt im Exil - in die Heimat zurückzukehren. Allein 2002 sollen nach UN-Angaben 1.834.537 Personen (davon 259.792 aus dem Iran und 1.565.066 aus Pakistan) zurückgekehrt sein. ${ }^{15}$

Auch in den folgenden Jahren remigrierten hunderttausende Afghan/inn/en, darunter einige tausend gebildete und oft gut situierte Personen, die nach Westeuropa oder die USA und nach Kanada geflohen waren und nun am Wiederaufbau des Landes mitwirken wollten (Houte 2016; Alam 2008; Rostami-Povery 2007).

Insgesamt dürften zwischen 2002 und 2014 rund 5,8 Mill. Afghan/inn/en zurückgekehrt sein, die meisten im Zeitraum bis 2005. Die Mehrzahl von ihnen, nämlich 3,9 Mill (902.000 aus dem Iran und ca. 3,8 Mill aus Pakistan), wurde mit Hilfe des UNHCR repatriiert, für das die Rückführung der afghanischen Flüchtlinge eine der größten Repatriierungsaktionen in der Geschichte der Organisation darstellte (MonsutTi 2008, p. 60).

Ab 2009 begannen die Rückkehrer/innenzahlen wegen der sich zunehmend verschlechternden Sicherheitslage zu sinken und erreichten 2014 - im Jahr des Abzugs eines großen Teils der internationalen Truppen - einen Tiefpunkt. Offiziellen Angaben zufolge migrierten 2014 nur 16.769 Personen nach Afghanistan zurück (OCHA 2015; WILLNER-REID 2017).

Erst seit einigen Jahren steigen die Rückkehrer/innenzahlen wieder stetig an. Verantwortlich dafür sind die immer restriktiveren Maßnahmen der iranischen und pakistanischen Regierungen und die sich verschlechternde Stimmung gegenüber afghanischen Flüchtlingen (siehe Ausführungen unten).

Allein aus Pakistan sollen laut den Angaben des „Baseline Mobility Assessments“ des IOM (2019b) zwischen 2016 und 2018 1.298.195 Afghan/inn/en und aus dem Iran 692.835 (im Sommer 1.991.030 Personen) mehr oder minder freiwillig zurückgekehrt sein. ${ }^{16}$

Die Migrationsbewegungen afghanischer Flüchtlinge in der Post-Taliban-Periode inkludieren jedoch nicht nur Rückkehrer/innen aus dem Exil, sondern umfassen auch Personen, die vor militärischen Auseinandersetzungen, ethnisch oder religiös motivierter Verfolgung oder aus anderen Gründen (z.B. Arbeitslosigkeit, Frustration über die politische Lage im Land, Problemen nach der Rückkehr in Afghanistan wieder Fuß zu fassen) innerhalb Afghanistans Schutz such(t)en oder ins Ausland flohen bzw. dorthin wieder zurückwanderten.

15 Vgl. im Detail CSO Afghanistan Statistical Yearbook 2011-2012; OCHA 2015.

16 Im Detail aus Pakistan: 2016: 766.907 Personen, 2017: 405.959 und 2018: 125.349; aus dem Iran 2016: 272.346, 2017: 241.105 und 2018: 179.384 Personen (IOM 2019b); Andere Organisationen, wie z.B. das UNHCR (2018b, p. 5), OCHA (2018a und 2018b) oder ECRE (2019, p. 4) nennen davon abweichende Zahlen. Vgl. auch NASEH et al. 2018; MaJid 2018; BJELICA 2016b, 2017 und 2018; Human Rights Watch 2017; Schmeidl 2019, p. 9; Grawert 2018; Grawert und Mielke 2018; Bezhan/Parsa 2018. 
Bereits kurz nach Beginn des als „Operation Enduring Freedom“ bezeichneten Antiterrorkampfes gegen Taliban und al-Qaida, der zunächst auf einige südliche und östliche Provinzen beschränkt blieb und erst später auf andere Landesteile ausgedehnt wurde, kam es zu umfangreichen neuen Fluchtbewegungen innerhalb Afghanistans und ins benachbarte Pakistan. Laut Angaben des UNHCR sollen allein bis Ende 2001 rund 150.000 Afghan/inn/en nach Pakistan geflohen sein. Weitere 1,2 Mill. sollen im Verlauf des Jahres 2002 im Ausland Schutz gesucht haben (Alam 2008, p. 16; SchmeidL 2019).

In den folgenden Jahren setzte sich dieser Trend fort. Bedingt durch die steigenden Zahl von Anschlägen und gewaltsamen Auseinandersetzungen zwischen Regierungstruppen und diversen Oppositionsgruppierungen (z.B. Taliban, Haqqani Netzwerk, Islamic State Khorasan Province/ISKP, Islamic Movement of Uzbekistan etc.) sowie Übergriffen krimineller Banden sahen sich ab 2009 immer mehr Afghan/inn/en zur Flucht genötigt. ${ }^{17}$

Diese durch Gewalt und Unsicherheit induzierte Mobilität wird beispielsweise an Hand der dramatisch gestiegenen IDP-Zahlen deutlich. Waren es 2009 „erst“ 297.0000 IDPs, so hat sich ihre Zahl laut Schätzungen von OCHA und UNHCR auf mittlerweile mindestens 2 Mill. (Stand Sept. 2018) erhöht (ECRE 2019 und SCHMEIDL 2019, p. 7). ${ }^{18}$

Allein für das Jahr 2018 nennt das „Baseline Mobility Assessment“ des IOM (2019b) 732.270 Personen, die infolge von Konflikten, Menschenrechtsvergehen, Dürre und anderen Naturkatastrophen zu IDPs wurden. ${ }^{19}$ Unter den in diversen IDP-Statistiken genannten Personen befinden sich zahlreiche Personen, die als Langzeit-IDPs gelten. Viele davon mussten zwei Mal oder sogar öfter fliehen (vgl. OCHA 2017 b).

Auch die Zahl der ins Ausland migrierenden Afghan/inn/en stieg wieder an. Angaben des UNHCR zufolge suchten zwischen 2015 und 2017 fast 1 Million (962.000) Afghan/inn/en weltweit um Asyl an, wobei die höchsten Zahlen in den Jahren 2016 (369.000) und 2017 (334.000) gemeldet wurden (vgl. SCHMEIDL 2019, p. 4). ${ }^{20}$

\footnotetext{
17 Insgesamt hat sich die Sicherheitslage in Afghanistan seit Ende 2014 deutlich verschlechtert. Gegenwärtig werden nur mehr rund 55,5 \% aller Distrikte von der Regierung kontrolliert, in 32,4 \% kämpfen Regierung und diverse aufständische Gruppierungen um die Kontrolle und 12 $\%$ werden von der Opposition (z.B. Taliban und ISKP) kontrolliert (vgl. SIGAR 2018, p. 70). Fast täglich kommt es zu Anschlägen. 2018 war bislang das Jahr mit den meisten zivilen Opfern (UNAMA 2019a und 2019b). Laut Angaben des OCHA 2019 kommt es in 32 der insgesamt 34 afghanischen Provinzen zu Vertreibungen.

Ad. Aktuelle Sicherheitslage in Afghanistan vgl. Clark 2019; Ecoi.Net 2018; SIGAR 2018, UNAMA 2019a, 2019b; RUTTIG 2018a und 2018b. Auf Grund der Eskalation gewaltsamer Auseinandersetzungen hat die UN Afghanistan 2017 von einem ,post-conflict-country“ zu einem Land ,in active conflict" reklassifiziert (OCHA 2017a).

18 Die Angaben zu den IDPs variieren sehr stark: Beispielsweise führte das IDMC (2018) rund 1,3 Mill. IDPs für 2017 an, während SAmuel Hall et al. (2018) von 1.948 .000 und das OCHA (2017a) sogar von rund 2,4 Mill. IDPs ausgingen.

${ }_{19}$ Laut Angaben des OCHA (2018c) sollen zwischen 2. Jänner und 8. Oktober 2018251.207 Personen auf Grund von Naturkatastrophen, primär wegen einer seit 2017 anhaltenden Dürre zu IDPs geworden sein (vgl. SCHMEIDL 2019, p. 4).

20 Davon abweichende Zahlen nennt das „Baseline Mobility Assessment“ des IOM. Dieses
} 
Bedingt durch die immer repressivere Flüchtlingspolitik ihrer Regierungen stellen die beiden jahrzehntlang als Hauptzielländer fungierenden Nachbarstaaten nur mehr mit Einschränkungen eine Zufluchtsoption dar, wie die Zahlen für beide Staaten illustrieren. Migrierten 2016 noch 373.950 Afghan/inn/en in den Iran, waren es 2017292.171 und 2018 nur mehr 204.277 Personen. Auch nach Pakistan wanderten deutlich weniger Personen, 2016 135.834, 201797.010 und 2018 lediglich 61.512 Afghan/inn/en (IOM 2019 b).

Für zahlreiche Afghan/inn/en nicht nur aus Afghanistan selbst, sondern auch für jene, die zuvor - oft viele Jahre im Iran oder in Pakistan gelebt haben oder sogar dort geboren wurden - sind Westeuropa (darunter auch Österreich) sowie die Türkei daher zu neuen alternativen Fluchtdestinationen avanciert (vgl. Donini et al. 2016, RIESENKAMPFF und NoOR EBAd 2017, HaKIMI 2016, p. 10). ${ }^{21}$

Dies veranschaulichen u.a. die in den letzten Jahren deutlich gestiegenen Asylantragszahlen. ${ }^{22}$ Waren es in den 1980er Jahren insgesamt nur 30.963 Afghan/inn/en (ALAM 2008, p. 17 nach Daten des UNHCR), die in ein europäisches Land flohen (im Vergleich dazu 5,5 bis 6,2 Mill. in den Iran und nach Pakistan), so hat sich ihre Zahl seit 2014 deutlich erhöht. Kamen 2014 erst 37.855 afghanische Asylwerber/innen in die EU, so stieg ihre Zahl 2015 bereits auf 178.230 Personen und 2016 - dem bislang antragsstärksten Jahr - sogar auf $186.595 .^{23}$

Mit der Schließung der Balkanroute im März 2016 begannen die Asylwerber/ innenzahlen wieder deutlich zu sinken. ${ }^{24}$ Laut Angaben von EUROSTAT stellten Afghan/ inn/en 2017 noch 43.600 Asylanträge in der EU, 2018 waren es nur mehr 41.000. ${ }^{25}$ In Österreich gingen die Antragszahlen von 25.475 (2017) auf 11.742 (2016), 3.676 (2017) und schließlich auf 1.734 im Jahr 2018 zurück (BM.I 2015, 2016, 2017, 2018). Dennoch zählen Afghan/inn/en neben Syrer/inne/n und Iraker/inne/n weiterhin zu den

führt für den Zeitraum 2015881.276 Out-Migrants an, ohne diese genauer zu spezifizieren. Für 2016 werden 604.998, für 2017475.286 und für 2018330.023 Personen genannt (IOM 2019b). 21 Von den laut UNHCR ca. 260.000 afghanischen Asylsuchenden im Jahr 2015 suchten laut EUROSTAT knapp zwei Drittel (181.400) Asyl in der EU (mehr als vier Mal so viele wie 2010 (SCHMEIDL 2019, p. 5).

22 Insgesamt verlief die Entwicklung der Asylantragszahlen nicht kontinuierlich und weist, was die einzelnen Staaten der EU angeht, zudem große Unterschiede auf. (vgl. Alam 2008, p. 17 ff.; Beller 2013, p. 36 ff.; Hofmann und Reichel 2014; Reichel und Hofmann 2011.

Ad. Afghanische Flüchtlinge in Europa (vgl. Gehrig 1999; Centlivres und Centlivres-Demont 2000; Braakman 2005; Tietjens 2002; Beller 2013; Hofmann und Reichel 2014; Houte 2016; Rutig 2017a, 2017b, 2017 c).

${ }_{23}$ Vgl. Houte 2016, p. 55, Tab. 22.; EUROSTAT Pressemitteilung 44/2016, 46/2017 und $47 / 2018$.

${ }_{24}$ Seither sitzen viele Afghan/inn/en, wie auch andere Asylwerber/innen in den Westbalkanländern fest vgl. Ruttig 2017 d; Wait 2028.

25 Vgl. EUROSTAT-Pressemitteilung 47/2018 vom 20. März 2018 und 46/2019 vom 14. März 2019. 
größten Gruppen, die in der EU einen Erstantrag stellen (EUROSTAT 2017 und 2018; vgl. SCHMEIDL 2019 und ECRE 2019, p. 1).

Was die geographische Verortung der afghanischen Asylwerber/innen angeht, so stellt die Bundesrepublik Deutschland für sie seit den 1980er Jahren das mit Abstand attraktivste Zielland in Europa dar. Neben Deutschland entwickelten sich einige andere europäische Staaten, darunter Österreich, Schweden, Belgien, Norwegen und die Niederlande, zu wichtigen Aufnahmeländern (vgl. Beller 2013; Alam 2008; Ruttig 2017a, b, c, d; Reichel und Hofmann 2011, p. 10; Hofmann und Reichel 2014, p. 107 f.; Daxner und Nicola 2017; Parusel 2018).

Parallel zu den EU-Staaten entwickelte sich die Türkei zu einem wichtigen Zielland für afghanische Asylwerber/innen und Migrant/inn/en. Nach Angaben des UNCHR sollen zwischen 2015 und 2017 insgesamt 363.000 Afghan/inn/en in der Republik Türkei um Asyl angesucht haben (vgl. SchmeIDL 2019).

Wie im Iran und in Pakistan so ist mittlerweile auch in der EU und in der Türkei der Druck auf Afghan/inn/en in die Heimat zurückzukehren deutlich angestiegen. Dies spiegelt sich u.a. in einer Reihe von Rückführungsabkommen zwischen der EU (z.B. das Joint Way Forward Agreement vom Oktober 2016) bzw. einzelnen europäischen Staaten (z.B. Deutschland, Finnland, Schweden) und der afghanischen Regierung sowie in vermehrten Ausreiseaufforderungen und steigenden freiwilligen und erzwungenen Rückkehrer/innenzahlen wider. ${ }^{26}$

Zwar liegen weder seitens des IOM noch der EU oder einzelner europäischer Staaten exakte Angaben zur Zahl der Rückkehrer/innen vor, dennoch deuten die verfügbaren Daten auf einen zahlenmäßigen Anstieg derselben hin. Während 2015 erst 3.300 Afghan/inn/en aus Europa in ihre Heimat zurückkehrten bzw. dorthin zurückgeführt wurden, waren es 2016 rund 9.600 und 2017 ca. 4.260 Personen. ${ }^{27}$

Auch die Türkei hat in den letzten Jahren begonnen, Afghan/inn/en zur Rückkehr in ihr Herkunftsland zu bewegen. So sollen laut Angaben von ECRE 2018 zwischen April und Anfang Mai rund 8.000 afghanische Staatsbürger/innen deportiert worden sein. ${ }^{28}$

\footnotetext{
${ }_{26}$ Das zwischen der EU und der afghanischen Regierung abgeschlossene „Joint Way Forward“Abkommen sieht die Rückkehr von rund 80.000 Afghan/inn/en in den nächsten Jahren vor. Im Gegenzug erhält die afghanische Regierung finanzielle Unterstützung, um eine geordnete Reintegration der Rückkehrer/innen zu bewerkstelligen (vgl. AMNESTY INTERNATIONAL 2017, p. 35 f.); ad. Rückführungen nach Afghanistan siehe AMNESTY INTERNATIONAL 2017; EUROSTAT-Statistics Explained 2019a; BJelica 2017, Parusel 2018; ad. Joint Way Forward siehe European Union External Action Service 2016.

27 Laut EU-Statistiken zitiert nach AmNesty International 2017, p. 31; Kepp et al. 2018, p. 7 und EUROSTAT-Statistics Explained 2019a. Das „Baseline Mobility Assessment“ des IOM 2019b, p. 3 nennt für 2016: 26.175, für 2017: 29.277 und für 2018: 29.812 Rückkehrer/innen aus Europa und der Türkei.

28 Der türkische Innenminister nannte in einer TV-Ansprache im Frühjahr 2018 sogar rund 15.000 Personen, die mit Charterflügen nach Afghanistan zurückgebracht wurden (vgl. VeRMA 2018).
} 


\section{Faktoren der rezenten Mobilität}

Nachdem die seit 1978/79 vonstattengehenden gewaltbedingten Migrationsbewegungen kurz dargestellt wurden, soll im Folgenden bezugnehmend auf rezente empirische Erhebungen unter afghanischen Flüchtlingen in Österreich die Bedeutung der Mobilität (insbesondere jener nach Europa) als Strategie der Überlebenssicherung beleuchtet werden und dabei auf einige sie beeinflussende Aspekte verwiesen werden: Denn wer wann und warum wohin migrierte bzw. migriert, hängt von einer Reihe unterschiedlicher Faktoren ab, wie z.B. der Einschätzung der Dauer einer Konfliktsituation, den vorhandenen ökonomischen Ressourcen, dem soziokulturellen Background, den bestehenden sozialen Netzwerken zu einem potentiellen Fluchtort und der aktuellen Asylpolitik in einem möglichen Zielland.

Die einzelnen Faktoren (z.B. Wahl des Zufluchtsortes, Migrationsgrund) stehen dabei häufig in einem engen Konnex zueinander und haben im Verlauf der letzten vier Jahrzehnte - wie die Ausführungen zu den vier Fluchtmigrationsperioden bereits illustriert haben - teilweise eine beträchtliche Veränderung ihrer Relevanz erfahren.

Ein illustratives Beispiel stellen hier z.B. die Hauptmotive für eine Migrationsentscheidung dar. Während anfänglich Flucht vor Krieg und politischer Verfolgung die Hauptgründe für eine Migration darstellten, ist es heute meist eine Kombination unterschiedlicher Faktoren, wie z.B. Verschlechterung der allgemeinen Sicherheitslage, erlittene Gewalt, Verfolgung wegen der Zugehörigkeit zu einer ethnischen oder religiösen Minderheit, ökonomische Ursachen, Frustration über die gegenwärtige politische Lage im Heimatland oder die Diskriminierung am gegenwärtigen Aufenthaltsort (z.B. im Iran), welche die Entscheidung zur Migration bzw. Weiterwanderung beeinflussen. ${ }^{29}$ Daneben spielen auch soziale Verpflichtungen gegenüber Angehörigen (z.B. der Wunsch das Leben von Familienangehörigen und Verwandten zu schützen oder den Kindern eine bessere Zukunft - Leben in Frieden, Bildungsmöglichkeiten - zu bieten sowie persönliche Gründe (z.B. Angst vor Zwangsverheiratung oder einer Vendetta) eine Rolle (RASUly-PALECZEK 2017).

Die Hauptgründe für eine Migration können dabei für die Angehörigen der verschiedenen afghanischen Bevölkerungsgruppen sehr unterschiedlich sein. Beispielsweise nannten zahlreiche von uns interviewte Paschtunen und Angehörige anderer ethnolinguistischer Gruppen (z.B. Tadschiken und Uzbeken) den Anstieg lokaler Gewalt und die häufig vorkommenden Selbstmordanschläge als primäres Motiv, während es für viele Hazara und andere Schiiten oft die Bedrohung durch die Taliban und in neuerer Zeit durch die ISKP (Islamic State Khorasan Province) ist, die sie zur Flucht bewegte (RAsuly-PALECZeK 2017, p. 65).

$29 \quad$ Allgemein ad. multiple Fluchtgründe: BrÜCKER et al. 2016, Crawley et al. 2016, Donini et al., LinKe 2016, FMR 2014, MaJidi 2018, Majidi et al. 2016, KaZemi 2016, Echavez et al. 2014; RASULY-PALECZEK 2017. 
Personen, die vor ihrer Flucht nach Österreich für afghanische Regierungsstellen oder ausländische Organisationen (z.B. als Übersetzer für die NATO-Truppen oder eine der in Afghanistan tätigen NGOs) gearbeitet haben, fühlen sich oft durch die Taliban, die ihnen die Zusammenarbeit mit den „Kufr“ (Ungläubigen) vorwerfen, bedroht (RAsuly-PaleczeK 2017, p. 65).

Für junge Afghan/inn/en der Mittelschicht, die in der Post-Taliban-Ära aufgewachsen waren und die trotz guter Ausbildung oft keine adäquate Beschäftigung finden, stellen wiederum Gefühle der Perspektivenlosigkeit und Frustration über die herrschenden politischen Verhältnisse und die im Land weitverbreitete Korruption einen wesentlichen Migrationsgrund dar (RASUly-PALECZEK 2017, p. 66; vgl. SAFi 2016).

Langzeitvertriebene und Rückkehrer/innen aus dem Iran und Pakistan motiviert meist das Fehlen der für einen Neustart erforderlichen finanziellen Mittel und sozialen Kontakte ${ }^{30}$ sowie die mangelhafte Unterstützung durch die afghanischen Behörden zu einer erneuten Migration (z.B. nach Europa). ${ }^{31}$ Auch die allgemein schlechte Wirtschaftslage, die zudem in einem engen Zusammenhang mit der prekären Sicherheitslage steht, ist hier von Bedeutung. ${ }^{32}$

Afghan/inn/en, die nicht direkt aus Afghanistan nach Europa migrieren, sondern aus Pakistan und dem Iran, wo sie lange Zeit gelebt haben bzw. sogar dort geboren wurden, ${ }^{33}$ oder die nach einem Aufenthalt in einem Transitland (z.B. der Türkei) in ein europäisches Land kommen, verweisen v.a. auf die sich in den letzten Jahren zunehmend verschlechternden Lebensbedingungen und die restriktive Asylpolitik ihrer früheren Asylländer.

Bei der Wahl eines Zufluchtsortes spielt ebenfalls eine Reihe von unterschiedlichen Faktoren eine Rolle. Relevant ist in diesem Zusammenhang zum einen die Einschätzung einer aktuellen Konfliktlage. Wird eine Bedrohung als nur von kurzer Dauer erachtet, so fliehen die Leute meist in das nächste als sicher geltende Gebiet (z.B. Nachbardorf, anderes Stadtviertel), wobei meist bei Verwandten oder Bekannten Schutz gesucht wird.

$30 \quad$ Vgl. Majidi 2018; Naseh et al. 2018; Ahmad 2019; Asylos 2017; Mixed Migration Centre 2019; Kepp et al. 2018; Grawert 2018; Grawert und Mielke 2018; Houte et al. 2015; Stahlmann 2017. Ad. Bedeutung sozialer Netzwerke: vgl. Berg Harpviken 2009; EASO 2018; Majidi 2018; Grawert und Mielke 2018. Das Fehlen sozialer Netzwerke in Afghanistan hindert viele im Iran oder Pakistan lebende Afghan/inn/en daran, eine Rückkehr dorthin ins Auge zu fassen (vgl. GrAWERT und MielKe 2018; MAJIDI 2018, SAITo 2007, 2009).

31 Vgl. Asylos 2017; AhMAD 2019; SIGAR 2015.

32 Vgl. Ahmad 2019; OCHA 2018 a, b, c. Laut Angaben der Weltbank leben ca. 39\% der Gesamtbevölkerung unter der Armutsgrenze (WORLDBANK 2017 zitiert nach OCHA 2017a). Rund 1,9 Mill. Afghan/inn/en gelten als „severly food insecure“ (OCHA 2017a). Der „Afghanistan Living Conditions Survey" veröffentlichte Daten, nach denen über die Hälfte der Bevölkerung unter der nationalen „Poverty Line“ leben muss (ECRE 2019, p. 2).

33 Rund $90 \%$ aller derzeit in Pakistan lebenden Afghan/inn/en wurden Schätzungen zufolge dort geboren (Grawert und Mielke 2018, p. 7; Schon 2005 waren mehr als 30\% aller dokumentierten Flüchtlinge im Iran Angehörige der 2. Generation (ABBASI-SHAVAZI et al. 2008, zitiert nach NASEH et al. 2018, p. 2). 
Bei einer länger andauernden Konfliktsituation wird oft das Gebiet verlassen und man flieht in eine andere Region (häufig in eine der großen Städte, die als sicherer gelten als der ländliche Raum) oder ins benachbarte Ausland. Heute ist dies, wie nachstehend ausgeführt wird, allerdings nur mehr sehr eingeschränkt möglich.

Zum anderen wird die Wahl des Zufluchtsortes von der Distanz zwischen aktuellem Aufenthaltsort und möglichem Migrationsziel sowie von schon bestehenden sozialen Netzwerken zum potentiellen Zufluchtsort determiniert. So migrierten beispielsweise die meisten afghanischen Dari-Sprecher/innen und Schiiten (darunter viele Hazara) ursprünglich v.a. in den Iran und nach Quetta/Pakistan, während sunnitische Muslim/ innen Pakistan bevorzugten, wobei die meisten paschtunischen Flüchtlinge primär in den dortigen Siedlungsgebieten der Paschtunen Zuflucht suchten. Afghanische Sikhs und Hindus flohen vor allem nach Indien, Angehörige des kommunistischen Regimes (ab 1989) in die Sowjetunion bzw. nach deren Zusammenbruch in die Russische Föderation, partiell auch nach Tadschikstan und Uzbekistan (vgl. BrAAKMAN 2005, p. 8 f.).

Daneben kommt den zur Verfügung stehenden ökonomischen Ressourcen und dem soziokulturellen Background der Migrant/inn/en eine erhebliche Bedeutung bei der Wahl des Ziellandes zu. Personen ländlicher Herkunft mit geringen finanziellen Mitteln flohen bis in die jüngste Vergangenheit in den Iran und nach Pakistan und verblieben auch dort. ${ }^{34}$

Angehörige der urbanen, vermögenderen und gebildeten tadschikischen und paschtunischen Elite und Mittelklasse, vor allem aus Kabul, migrierten entweder direkt ins westliche Ausland oder flohen nach einem kurzen Zwischenstopp im Iran oder Pakistan in den Westen, wobei die reichsten oft in die USA und nach Kanada gingen, während Angehörige der Mittelklasse meist in Europa blieben (vgl. BraAKMAN 2005, p. 9). Bei der Auswahl ihres jeweiligen Fluchtlandes spielten familiäre Kontakte (z.B. Verwandte, die schon früher in einem bestimmten europäischen Land lebten), frühere Aufenthalte (z.B. Studienzeit an einer Universität im Ausland) sowie die Asylpolitik des potentiellen Aufnahmelandes eine Rolle.

Auch bezüglich ihres religiösen und ethnolinguistischen Backgrounds ${ }^{35}$ unterscheiden sich die heutigen afghanischen Asylsuchenden von den früher geflohenen. Waren es anfänglich v.a. sunnitische Dari-Sprecher (z.B. Tadschiken, Kabuli ${ }^{36}$ und iranisierte Paschtunen sowie einige schiitische Hazara) die in Europa Schutz suchten, so befinden sich unter den rezenten afghanischen Asylwerber/inne/n Angehörige aller eth-

34 Heute kommen auch Personen aus diesen Bevölkerungsgruppen zunehmend nach Europa.

35 Zum Anteil der verschiedenen ethnolinguistischen und religiösen Gruppen liegen keine Angaben vor, zumal diese in den öffentlich zugänglichen Quellen (z.B. EUROSTAT, BMI-Statistiken) nicht angeführt werden.

36 Die Einwohner/innen Kabuls werden in Afghanistan meist mit dem Sammelbegriff „Kabuli“ bezeichnet, ohne dass eine Differenzierung nach der ethnolinguistischen Zugehörigkeit vorgenommen wird. 
nolinguistischen Gruppen (z.B. auch Turkmenen, Uzbeken etc.), wobei insbesondere die Hazara einen sehr hohen Anteil stellen dürften (SADAT 2017). ${ }^{37}$

Insgesamt kam es im Lauf der Zeit zu einer Schwerpunktverlagerung bei der Wahl der Zielorte (vgl. u.a. die Ausführungen zu den vier Fluchtmigrationsperioden). Suchten bis zum Ende der Taliban-Herrschaft (2001) fast ausschließlich Personen, die der urbanen Ober- und Mittelschicht angehörten und meist eine gute Schul- und Berufsausbildung hatten, Zuflucht in westlichen Ländern, so ist der sozioökonomische Background der heute nach Europa kommenden afghanischen Asylwerber/innen wesentlich komplexer und umfasst zunehmend auch Personen aus der urbanen Unterschicht oder aus dem ländlichen Raum, die oft über keine oder nur eine rudimentäre Schul- und Berufsausbildung verfügen. ${ }^{38}$ Darunter befinden sich auch zahlreiche Personen (vor allem in der Gruppe der UMF), die nicht direkt aus Afghanistan migrierten, sondern zuvor schon längere Zeit in Pakistan oder Iran gelebt hatten oder sogar dort geboren worden sind.

Hauptverantwortlich für die zunehmende Attraktivität Europas - auch für ärmere Afghan/inn/en - ist die Asylpolitik der klassischen Aufnahmeländer Iran und Pakistan sowie des Transitlandes Türkei. Diese ist seit 1978/79 deutlich restriktiver geworden.

Während anfänglich die zu hunderttausenden ins Land strömenden afghanischen Flüchtlinge positiv aufgenommen wurden und großzügige Unterstützung durch die lokale Bevölkerung, die Regierungen sowie im Falle Pakistans auch seitens einer Vielzahl an NGOs, erhalten haben, hat sich die Lage der Afghan/inn/en seit dem Ende des kommunistischen Regimes (April 1992) zunehmend verschlechtert.

Sowohl die iranische wie auch die pakistanische Regierung forderten die Flüchtlinge zur Rückkehr in ihre Heimat auf und begannen ihre Unterstützungsprogramme für Flüchtlinge zu reduzieren. Beispielsweise schloss Pakistan zahlreiche Flüchtlingslager und versuchte weitere, vor den Konflikten zwischen den Mujaheddin-Fraktionen bzw. den Taliban-Repressionen fliehende Personen am Grenzübertritt zu hindern (RASUlYPaleczer 2001). Zudem waren afghanische Flüchtlinge mit steigender Fremdenfeindlichkeit konfrontiert. Sie wurden von der Lokalbevölkerung zunehmend als ökonomische und soziale Bürde betrachtet und für eine Reihe von Problemen (z.B. Arbeitslosigkeit, ausufernder Drogenhandel, Anstieg von Gewalt und Kriminalität) verantwortlich gemacht. Ihre Fluchtgründe - Angst vor Verfolgung und Krieg - wurden umgedeutet zu ökonomisch motivierter Zuwanderung und die Flüchtlinge und Asylwerber/innen als illegale Immigrant/inn/en stigmatisiert.

\footnotetext{
$37 \quad$ Viele rezente afghanische Flüchtlinge in Europa dürften ursprünglich im Iran gelebt haben oder dort geboren worden sein (vgl. SADAT 2017 und unsere Interviewdaten). Die offiziellen Asylstatistiken liefert dazu allerdings keine Informationen.

38 RiesenKampFF und Noor ABAD (2016, p. 1 f.) verweisen auf eine Studie des deutschen Bundesamts für Migration und Flüchtlinge (BAMF): „Demzufolge haben mittlerweile $26 \%$ der afghanischen Asylbewerber überhaupt keine Schule besucht, und lediglich $28 \%$ verfügen über einen mittleren oder höheren Schulabschluss.“
} 
Nach dem Ende des Taliban-Regimes verschärfte sich die Lage der Flüchtlinge noch weiter. Mit verstärkten Grenzkontrollen, verminderten Hilfsangeboten und vielfältigen administrativen Schikanen versuchen die beiden afghanischen Nachbarstaaten potentielle Flüchtlinge am Kommen zu hindern bzw. zu einer Rückkehr in die Heimat zu veranlassen. ${ }^{39}$

Ähnlich problematisch ist die gegenwärtige Lage vieler afghanischer Flüchtlinge in der Türkei. Sie stellt für Afghan/inn/en heute kein alternatives Asyl- oder sicheres Transitland mehr dar. ${ }^{40}$ Während die türkische Regierung Anfang der 1980er Jahre noch mehrere tausend Angehörige der afghanischen Turkvölker (z.B. Uzbeken und Kirgisen) aus pakistanischen Flüchtlingslagern in die Türkei brachte (SEYHAN 2017) und in die türkische Gesellschaft integriert hat, sind afghanische Flüchtlinge heute nicht mehr willkommen. Im Unterschied zu syrischen Flüchtlingen, die seitens der türkischen Behörden eine gewisse Unterstützung erhalten (z.B. Gesundheitsversorgung, Bildungszugang etc., siehe Türkei-EU-Deal), bekommen die 157.000 afghanischen Flüchtlinge (VERMA 2018) keinerlei offizielle Unterstützung und sind auf die humanitäre Hilfe durch türkische Privatpersonen und NGOs angewiesen. Mittlerweile hat auch die Türkei mit der erzwungenen Rückführung afghanischer Flüchtlinge begonnen (ECRE 2018 und VERMA 2018).

Insgesamt wird es - wie die vorangegangenen Ausführungen darlegten - für Afghan/inn/en immer schwieriger, ein Zielland für ihre Migrationswünsche zu finden. Sowohl die beiden ,klassischen“ Destinationen Iran und Pakistan wie auch später Europa und die Türkei stellen heute nur mehr sehr bedingt eine Migrationsoption dar, wie auch einer unserer Respondent/inn/en feststellte:

„Dann sag“ ich, wo können wir Afghanen noch hingehen? Flüchten von Afghanistan nach Pakistan. Pakistan wird uns auch töten, wird uns umbringen, wird es mit uns machen wie Al Quaida. Dann gehen wir in den Iran und dann sagen unsere muslimischen Brüder, ..., die sind auch Schiiten .... Und die sind genauso wie alle anderen. Dann kommen wir in die Türkei. Die Türkei schiebt uns zurück in den Iran. Das heißt, wir sind so wie ein Fußball ohne Luft, einfach. Ja, dich braucht man nicht, mit dir kann man nicht spielen. Das heißt, wir werden überall gefoltert, umgebracht, getötet, aber die Welt schaut einfach zu. " (I 27, männlich, 27 Jahre alt)

$39 \quad$ Ad. Situation afghanischer Flüchtlinge im Iran und in Pakistan sowie aktuelle Asylpolitik der beiden Staaten siehe Ahmadi 2015; Kabir 2015; Giles 2010; Hoodfar 2010; Abbasi 2015; WiLlNER-Reid 2017; Majidi et al. 2016; Majidi 2018; Human Rights Watch 2013; Rasuly-PaleczeK 2017; Adel-Krah und Olszewska 2007; AbBasi-Shavasi und GlazenbrooK 2006; BJelica 2016a und 2018, Ahmad und Fanizadeh 2018; Alima 2018; Zieck 2008; Kronefeld 2008; Human Rights Watch 2017; Schmeidl 2019; Grawert und Mielke 2018; Naseh et al. 2018; Grawert 2018. Ad. Repatriierungen aus Iran und Pakistan siehe Ausführungen oben.

40 Ad. Situation afghanischer Flüchtlinge in der Türkei vgl. Ajam Media Collective 2015; Arjomand 2016; Cheslow 2016; Kayaoglu 2015; Picozza 2017; Kaytaz 2016; Ozlati-MoghadDAM 2012. 


\section{Multiple Wanderungen/Migrationen als Überlebens- strategie}

Schon vor dem Einsetzen der kriegsbedingten Migrationsbewegungen waren große Teile der afghanischen Bevölkerung äußerst mobil (MonsutTi 2005, 2008; GRAWERT und MielKe 2018). Als Folge der seit vier Jahrzehnten andauernden Konflikte hat die Mobilität der Afghan/inn/en jedoch ein Ausmaß erreicht, welches die afghanische Bevölkerung nachhaltig geprägt hat. Jeder vierte der derzeit rund 34 Mill. Afghan/inn/ en soll Angaben des IOM (2019a) zufolge zumindest einmal in seinem Leben vertrieben worden sein. Noch höhere Zahlen nennt SchmeIdL (2019, p. 3) Bezugnehmend auf eine von Oxfam, dem Internationalen Roten Kreuz gemeinsam mit mehreren afghanischen NGOs durchgeführte Befragung verweist sie darauf, dass vor der letzten großen Vertreibungswelle, die 2015 begann, ,... jeder zweite Afghane mindestens eine (viele sogar mehrfache) Vertreibungserfahrung(en) gemacht [hatte].“41

Durch Vertreibung oder wirtschaftliche Notlage erzwungene Mobilität (sowohl innerhalb Afghanistans wie auch ins Ausland bzw. zwischen dem Ausland und Afghanistan) ist mittlerweile für große Teile der afghanischen Bevölkerung zur Regel und zu einer wesentlichen Überlebensstrategie geworden (SCHMEIDL 2019, p. 1; MonsutTI 2008, p. 59 und 61).

Die gilt ebenso für zahlreiche rezente afghanische Asylwerber/innen, deren Leben schon bevor sie nach Europa kamen durch multiple Mobilitätserfahrungen geprägt war. Sie waren zuvor IDPs oder Rückkehrer/innen nach Afghanistan oder haben länger in den beiden Nachbarstaaten Iran und Pakistan gelebt oder wurden sogar dort geboren bzw. haben eine längere Zeitspanne in einem Transitland (z.B. Türkei) verbracht, um Geld für die Weiterreise zu verdienen. ${ }^{42}$

Dies belegen auch die von uns durchgeführten Erhebungen. Beispielsweise befand sich fast die Hälfte der 65 Befragten der „Afghan/inn/en-Studie“ vor ihrer Ankunft in Österreich längere Zeit im Iran (insgesamt 18 Personen) oder in Pakistan (11 Personen) oder war dort geboren worden (vgl. FRIEDRICH et al. 2019).

Insgesamt ergaben unsere Befragungen ein sehr komplexes Bild der Mobilitätsstrategien der nun in Österreich lebenden Afghan/inn/en.. ${ }^{43}$ Viele unserer Respondent/ inn/en erwähnten, dass sie vor ihrer Migration nach Österreich zunächst innerhalb Afghanistans an einem sicheren Ort Schutz suchten und erst später, als ihnen ein Ver-

\footnotetext{
„The study concluded that $76 \%$ of respondents had been forced to leave their homes at some point. Of these, $41 \%$ were internally displaced, $42 \%$ were externally displaced and $17 \%$ had been both internally and externally displaced (Oxfam, 2009) Many individuals were displaced multiple times." Hakimi (2016, p. 8 f.), der auf die gleiche Studie wie SchmeIDL verweist.

${ }_{42}$ Die offiziell zugänglichen Daten liefern dazu aber keine aussagekräftigen Informationen.

43 Vgl. die in Grawert und MielKe 2018 angeführten Fallbeispiele.
} 
bleib in Afghanistan zu unsicher erschien, in eines der Nachbarländer geflohen sind und von dort dann - oft nach längerem Aufenthalt - nach Österreich weiterreisten:

„... als ich in der ersten Schulstufe war, sind die Taliban gekommen und es ist alles durcheinandergeraten. Jede/r ist geflüchtet. Wir sind nach Panjshir [eine Provinz] gegangen, von dort aus sind wir nach Pakistan gegangen. Und dann als wir zurück waren, war auch die wirtschaftliche Lage schlecht. Es war niemand, der/die dort arbeiten konnte, dann sind wir wieder nach Afghanistan gekommen ...." (I 58, weiblich, 31 Jahre alt)

Andere migrierten von Afghanistan direkt in den Iran oder nach Pakistan, verbrachten dort viele Jahre im Exil und kamen schließlich wegen der zunehmend schwierigeren Lebensbedingungen und der sich verschärfenden Flüchtlingspolitik ihrer früheren Aufnahmeländer nach Österreich. Ein 25jähriger Hazara, der als Kind mit seiner Familie wegen der Verfolgung durch die Taliban nach Iran floh, beschrieb die Situation afghanischer Flüchtlinge wie folgt:

„, [...] Taliban, deshalb hat mein Vater beschlossen, in den Iran zu flüchten. Angekommen im Iran, haben wir rund 21 Jahre dort gelebt, hatten ein eigenes Leben. .... Ja, die Jahre vergingen, dann hat die iranische Regierung beschlossen, den Afghanen den Zugang zur Bildung nicht mehr zu gewähren. .... Naja, die Afghanen im Iran haben es nicht besonders leicht, sowohl die, die längere Zeit dort leben, als auch die Neuankömmlinge. ... Ein Afghane muss bis zu seinem Tod arbeiten, er ist nicht versichert, er muss sich die Schule selbst finanzieren, die Medikamente selbst finanzieren! Ja, ein Afghane ist dort wie ein Tier, besitzt keine Rechte. Es wird nicht gesagt, ja, das sind auch Menschen, nehmen wir ihre Hand und helfen ihnen [...]. " (A 5, männlich, 25 Jahre)

Ähnlich schwierig war die Situation in Pakistan, wie eine junge Afghanin erläuterte:

„,Da wir in Pakistan gelebt haben und keine Ausweise hatten, und jene Personen, die über keine Papiere verfügten, zurück nach Afghanistan geschickt wurden, und wir auch keine Familie und keinen Platz (Zuhause) in Afghanistan hatten ... Wenn wir zum Beispiel zurückgegangen wären, hätten wir keine Arbeit gehabt. Aus diesem Grund sind wir nicht in Pakistan geblieben und sind nach Österreich gekommen. (lacht) “(I 61, weiblich, 25 Jahre)

Wieder andere kehrten nach einem längeren Aufenthalt in Iran oder Pakistan zunächst nach Afghanistan zurück oder wurden dorthin deportiert. Sie entschlossen sich 
aber aus unterschiedlichen Gründen (schlechte Sicherheitslage, wirtschaftliche Situation oder der Unmöglichkeit in der Heimat Fuß zu fassen) zu einer neuerlichen Migration, diesmal nach Österreich.

So etwa im Fall von A 18. Er wurde im Iran geboren, wohin sein Vater, der in Moskau studiert hatte, während der sowjetischen Okkupation Afghanistans (1979-1989) geflohen war. Im Iran musste dieser den Unterhalt der Familie als einfacher Arbeiter verdienen. Während der Amtszeit von Präsident Karzai (Ende 2001 bis September 2011) kehrte die Familie, wie Millionen anderer Afghan/inn/en, in die Heimat zurück. A 18 fand sich in Afghanistan nicht zurecht ,... Afghanistan war nicht, was ich erwartet hatte, die Kluft zwischen Arm und Reich war sehr groß .... Ich möchte Afghanistan nicht, das Land war auch nicht sicher ... " (A18, männlich, 23 Jahre). A18 lebte ein Jahr mit seinen Eltern und entschied sich dann dafür in den Iran zurückzukehren. Wegen der zunehmenden Diskriminierung afghanischer Flüchtlinge konnte er dort keine Arbeit finden. Gemeinsam mit einem Freund verließ er schließlich den Iran und machte sich auf den Weg nach Europa.

Auch im Falle von A 19 war die Remigration nach Afghanistan nur von kurzer Dauer. Seine Familie war 1986 vor den Taliban nach Pakistan geflohen, wo er die Schule absolvierte. 2003 kehrte die Familie in die Heimat zurück. A 19 arbeitete zunächst in einem afghanischen Ministerium und später für eine US-Sicherheitsfirma. Während dieser Zeit absolvierte er mit US-Unterstützung außerdem ein Studium in Großbritannien. Aufgrund seiner Tätigkeit für ausländische Firmen erhielt A 19 immer wieder Drohungen. Diese bewogen ihn zunächst dazu mehrfach den Arbeitsplatz zu wechseln. 2013 wurde ein Attentat auf ihn verübt, was ihn letztendlich veranlasste aus Afghanistan zu fliehen. Außer A19 flohen noch andere Mitglieder seiner Familie. So sein Bruder, der als Journalist tätig war und ebenfalls bedroht wurde. Er gelangt in die USA und konnte später Teile der Familie nachholen.

Bisweilen haben unsere Respondent/inn/en auch mehrfach ihre Zielländer gewechselt.

So etwa im Fall von I 3. Als sie 4 Jahr alt war, floh ihre Familie zunächst nach Pakistan, wo sie sich rund ein halbes Jahr aufhielten. Von dort migrierten sie dann in die Ukraine, wo der Vater noch heute lebt. Die Mutter kam schließlich mit I 3 und deren Schwester nach Österreich.

Die Familie von A 20 suchte, als dieser 7 Jahre alt war, nachdem ihre Herkunftsregion von den Taliban erobert worden war, Schutz im Iran. A 20 verließ später seine Familie und migrierte nach Europa:

„,... ich habe meine Eltern verlassen und bin in die Türkei gegangen. Ein Jahr war ich in der Türkei, danach war ich drei Jahre in Griechenland. Nach drei Jahren habe ich die Chance ergriffen und bin hierhergekommen nach Österreich; wollte von hier nach Deutschland. In München wurde ich angehalten und sie haben mich 
zurückgeschickt. Sie haben mir gesagt, dass Österreich besser sei und so, und wir sind hier geblieben, wir waren schlussendlich in einem Camp. " (A 20, männlich, 24 Jahre)

Das Pendeln zwischen Afghanistan und einem der Nachbarländer oder die Migration zwischen Iran und Pakistan wurden gleichfalls als Teil der multiplen Mobilitätserfahrungen genannt, wie das Beispiel der Familie von A 17 demonstriert. Sie floh während des Taliban-Regimes (1985-2001) zunächst nach Quetta in Pakistan. Der Vater von A 17 ging dann zum Arbeiten in den Iran. Später folgte ihm die Familie, da sie sich als Schiit/inn/en in Pakistan diskriminiert fühlten. Im Iran lebten sie in schwierigen wirtschaftlichen Verhältnissen, was den Vater schließlich dazu bewog A 17 wegzuschicken, damit dieser eine bessere Zukunft haben würde. Nach zahlreichen Problemen kam A 17, der damals noch minderjährig war, schließlich nach Österreich. ${ }^{44}$ Seine Familie lebt mittlerweile in Australien:

„A17: In Pakistan vier Jahre. Wir haben zum Beispiel die Sprache gelernt. Es war gut. Dort gab es dasselbe Problem, das religiöse Problem, dass wir Schiiten waren. Sie wissen das bestimmt, in Quetta wird man sehr diskriminiert. Das war ein Verbrechen, dass wir Hazaras waren und zweitens, dass wir Schiiten waren .... Ja, mein Vater hat auch die Lage im Iran gesehen. Er konnte dort nicht leben. Zuallererst will ein Vater den Sohn nicht wegschicken. Mein Vater ist sehr gebildet, ich bin als Kind hierhergekommen. Der Iran ist nicht unschuldig. Sie diskriminieren und ärgern die Afghanen ... Mein Vater hat nachgedacht ..., das Geld zusammengestellt und gesagt, geh!'. Ich weiß nicht wie, aber er hat mich weggeschickt, ...Ich war neun bis zehn Monate auf dem Weg, war mit sehr vielen Problemen konfrontiert, ... “ (A 17, männlich, 21 Jahre)

Fehlende Ressourcen und Kontakte in Afghanistan verhindern oft eine Rückkehr in die Heimat und führen nach der Remigration dorthin dazu, dass eine erneute Migration, diesmal nach Europa stattfindet, wobei diese oft mit mehreren Zwischenstopps erfolgt. ${ }^{45}$ Dies illustrieren die nachfolgenden Aussagen unserer Respondent/inn/en:

\footnotetext{
44 Oftmals werden Kinder und Jugendliche von ihren Familien nach Europa geschickt, um ihnen eine bessere Zukunft zu ermöglich oder über eine spätere Familienzusammenführung selbst nach Europa nachkommen zu können (vgl. NicoLINI 2013; UNHCR 2010; LinKe 2016; FosCHINI 2017; WILKENs 2016). Allein zwischen 2008 und 2016 haben mehr als 100.000 afghanische UMFs in der Europäischen Union um Asyl angesucht (FoschinI 2001, p. 1); ad. Asylanträge von UMF in der EU vgl. EUROSTAT Newsrelease 2017 und EUROSTAT Datenset 2018.

${ }_{45}$ Oft kommt es nach der Rückkehr nach Afghanistan zu einem „Secondary Displacement“. Besonders davon betroffen sind arme Rückkehrer/innen und Personen, die im Exil geboren wurden (vgl. Grawert 2018, Grawert und Mielke 2018, Monsutti 2008, Madjidi 2018, Naseh et al. 2018).
} 
„Angesichts der vielen Probleme und Schwierigkeiten hat die iranische Regierung unsere Ausweise für ungültig erklärt und uns nach Afghanistan abgeschoben. In Afghanistan war ich gezwungen, nach Europa zu flüchten. " (I 49, männlich, 22 Jahre)

„, B: Als ich nach Österreich gekommen bin, da wollte ich nur ... Erstens, in unserem Land ist es ja nicht ruhig. Ich habe Afghanistan nie gesehen, ich bin im Iran geboren. Und ich hatte immer große Angst vor Afghanistan. Als mein Mann gesagt hat, dass wir in die Türkei fahren sollen, habe ich ihm zugestimmt.

Und als wir in der Türkei waren, hat sich das eine Jahr wie die Hölle auf Erden für mich angefühlt. Das eine Jahr hat sich wie 100 Jahre angefühlt. Wir hatten weder Gas noch Licht, damit wir das Haus aufheizen konnten. Wir mussten immer Feuer machen. Kohle und Steine und Schmutz ... Alles war einfach furchtbar!

Und als alle Richtung Europa gefahren sind, dann hieß es wieder: ,Gehen wir, gehen, wir, gehen wir, gehen wir! " Dann haben wir uns Geld ausgeborgt, um nach Europa zu kommen. Nur für die Kinder haben wir das gemacht. Nur, damit unsere Kinder nicht eines Tages wie ihr Vater ein Bauarbeiter werden, damit Sie eine bessere Zukunft haben und mehr erreichen können in ihrem Leben. Und dann ... die Sicherheit! Es ist wichtig, dass wenn man irgendwo lebt und sich nachts hinlegt, dass man sich sicher fühlt. " (I 10, weiblich, 29 Jahre)

„... also kann ich nicht hier leben. Ist mir egal wo, ehrlich! Wollte einfach weg von diesem Land [gemeint ist der Iran]. Und Afghanistan, ja! Ich habe sicherlich keine Chance gehabt, weil ich nicht mal Afghanistan gesehen habe, .... Und ich habe keine Kontakte in Afghanistan gehabt. Also offiziell ich komme aus Afghanistan, aber praktisch ich komme nicht aus Afghanistan. Weil ich niemand, nichts von dort kenne. OK? So bin ich geflüchtet vom Iran aus. Ich war in der Türkei, ich war in Griechenland, war so unterwegs und ich bin in Österreich geblieben, ich weiß nicht warum. " (I 11, männlich, 29 Jahre)

Bisweilen hatten einzelne Personen, bevor sie schließlich nach Österreich gelangten, bereits eine sehr komplexe Fluchtgeschichte durchlebt, wie das nachstehende Beispiel illustriert. Hier floh die Familie von I 27 zunächst nach Pakistan. Sein Vater war dann längere Zeit als Arbeitsmigrant in Saudi-Arabien, um den Unterhalt der Familie zu verdienen. Während dieser Zeit wollte I 27 gemeinsam mit seinem Cousin über den Iran nach Europa fliehen, wurde von den iranischen Behörden jedoch verhaftet und nach Afghanistan abgeschoben. Von dort kehrte er dann zu seiner in Pakistan lebenden Familie zurück. Der Vater floh später nach Österreich und konnte nach einiger Zeit die Familie nachholen. 
„Ich erzähle ... persönlich. Wir sind von Afghanistan geflüchtet ... Ende 1993 wir sind nach Pakistan gekommen, weil mein Vater natürlich in Afghanistan Probleme hatte. In Pakistan ich habe meine Schule fertiggemacht. Ich habe natürlich selber ein gutes Leben gehabt dadurch, dass mein Vater nicht mehr zurückgehen konnte. Mein Vater war in Saudi-Arabien bis Zweitausend ... . Zweitausend ist mein Vater nach Europa ge- kommen. 2001 in Österreich angekommen. Und Vater hat uns alle nachgeholt. Aber davor, 2003, ich war natürlich sehr jung, mein Cousin hat gesagt: , Gemma nach Europa!' Einmal versucht, mit Schlepper von Pakistan nach Iran zu kommen und von Iran nach Türkei und Türkei halt Richtung Europa. Leider Gottes bin ich im Iran verhaftet worden. Damals war ich 12, 13. Einmal war ich in einem der gefährlichsten Gefängnisse im Iran. Heißt ... [unverständlich]. Und dann haben die uns abgeschoben nach Afghanistan. Dadurch, dass ich von Afghanistan nach Pakistan gekommen bin, ich war ein kleines Kind. Ich wusste nicht, was ich da mache. Irgendwie bin ich zurück nach Pakistan gekommen. Das war 2003 [unverständlich] und 2005 hat mein Vater das Visum geschickt und wir sind mit Familie und Geschwistern nach Europa gekommen. " (I 27, männlich, 28 Jahre)

Und schließlich kann die Flucht eines Familienangehörigen auch die Notwendigkeit zur Mobilität anderer Familienmitglieder nach sich ziehen, wie die beiden nachfolgenden Beispiele aufzeigen.

A 12 (männlich, ca. 50 Jahre) musste wegen Drohungen Afghanistan verlassen und floh über Iran nach Österreich. Seine Frau und seine Kinder migrierten dann nach Pakistan, wo sie beim Vater der Frau lebten, bis A 12 Asyl erhielt und die Familie nachholen konnte.

Besonders illustrativ ist das nachfolgende Beispiel von I 15, der gemeinsam mit seiner Mutter und seinen Geschwistern mehrfach den Aufenthaltsort wechselte, bis eine Familienzusammenführung schließlich die Einreise in Österreich ermöglichte:

„, Wir sind nach Pakistan zuerst mal geflüchtet und von Pakistan dann ..., weil mein Vater war schon in Österreich und er hat auch Asyl bekommen und dann gibt es ja ... I: Familienzusammenführung! I 15: Weil wir [gemeint ist Informant und seine Mutter und Geschwister] haben neun Monate auch im Iran gelebt. I: Also, ihr seid von Afghanistan nach Pakistan ... I 15: ... von Pakistan nach Iran. ... Weil ein Onkel von mir war dort, und er hat uns auch geholfen mit dem ganzen Papierkram. Mein Vater ist dann später uns sozusagen abholen gekommen. ... “ (I 15, weiblich, 27 Jahre) 


\section{Resumee}

Bedingt durch die lange Konfliktdauer haben Millionen von Afghan/inn/en mehrfach Displacement, Migration und Neuanfang in einer für sie fremden und oft ablehnenden Umgebung erlebt. Multiple Mobilitätsformen, Exil und translokale Kontakte zu Verwandten und Bekannten, die über die ganze Welt verstreut leben, sind mittlerweile zu einem fixen Bestandteil ihrer Alltagserfahrung geworden, wie auch die nachstehende Aussage eines jungen Afghanen verdeutlicht:

„Ich will in Afghanistan leben. Dort lassen uns die Taliban, Terroristen, IS, Präsident, alle möglichen nicht leben. Dann komme ich nach Pakistan, da lässt man mich trotzdem nicht leben. Dann komme ich in den Iran, da lässt man mich auch nicht leben. Komme ich nach Europa, schicken mich alle zurück. 80\% der Afghanen, die in Afghanistan leben, sogar mehr als 80\%, haben nie die Liebe von Eltern erlebt - bis jetzt. Nicht einmal den Kopf an eine Mutterschulter angelehnt, weil die nie zuhause sind, die sind immer auf der Flucht. .... Heiraten heute, nach drei Tage ist Krieg. Dann musst du wieder nach Pakistan gehen und Geld verdienen, damit die Frau leben kann. Ich meine ist das ein Mensch? Das werden die Menschen nicht einmal eine Woche aushalten, den Mann nicht zu sehen und seine Tochter oder Kinder. Wir halten jahrelang durch, ... 20, 30 Jahre lang. Ich bin auf die Welt gekommen, ich hab ' mein Vater dann gesehen, als ich neun Jahre alt war. Ich meine, das ist nur meine Geschichte, aber es ist für hunderte Menschen, tausende Menschen so. " (I 27, männlich, 28 Jahre)

Die Motive für eine Migrationsentscheidung haben sich dabei im Lauf der Zeit verändert. Während es in der Ära der VDPA-, Mujaheddin- und Taliban-Herrschaft primär politische Gründe waren, die zur Migration in die Nachbarländer Iran und Pakistan oder nach Europa und Nordamerika zwangen, ist es heute eine Kombination unterschiedlicher Faktoren, die nun relevant ist. Neben der Verschlechterung der allgemeinen Sicherheitslage oder erlittener Gewalt beeinflussen zunehmend auch andere Motive, wie z.B. schlechte ökonomische Lage, Frustration über die politische Situation in Afghanistan, Unmöglichkeit, sich nach der Rückkehr in der Heimat ein neues Leben aufzubauen, sowie Diskriminierung und Asylpolitik am gegenwärtigen Aufenthaltsort (z.B. Iran und Pakistan), die Entscheidung zur Migration oder Weiterwanderungen.

Das Leben zahlreicher Afghan/inn/en ist - wie auch unsere Erhebungen unter in Österreich lebenden afghanischen Asylwerber/inn/en und Aslyberechtigten ergaben durch multiple Mobilitätserfahrungen (z.B. als IDP, Flüchtling oder Arbeitsmigrant/in Iran und Pakistan, Rückkehrer/in oder Remigrant/in) geprägt bzw. wie MonsutTI (2008, p. 59) formuliert „,The leaving and coming has been constant.“ Und „Families and individuals continue to move, and it seems unlikely that the back- and forth movements will 
stop while they constitute a key livelihoods strategy. Many Afghans have been shifting from one place to the next for years - some never returning to their place of origin, others only on a temporary basis before deciding to return into Iran, Pakistan or further afield.“ (MonsutTi 2008, p. 61).

Wer, wann und warum migriert bzw. migrierte hängt somit sowohl von den politischen Verhältnissen (z.B. Krieg, persönliche Bedrohung), wie auch von ökonomischen Faktoren (z.B. Arbeitslosigkeit in Afghanistan, wirtschaftliche Not im Flüchtlingslager) und persönlichen Gründen (Gefühl der Perspektivenlosigkeit, politische Frustration, Angst vor Zwangsverheiratung etc.) ab. Insgesamt ist eine Fluchtmigration häufig auch eng mit der Notwendigkeit einer Arbeitsmigration verknüpft. Die schwierigen Lebensbedingungen an einem aktuellen Aufenthaltsort (z.B. Flüchtlingslager) machen es oft erforderlich, dass einzelne Familienmitglieder anderswo Arbeit suchen, um dadurch das Leben ihrer Angehörigen im Camp zu sichern. Beispielsweise migrierten schon den 1990er Jahren viele afghanische Männer aus den Flüchtlingslagern im Gebiet von Peschawar oder Haripur nach Karachi, um dort permanent oder saisonal zu arbeiten. ${ }^{46}$

Zahlreiche empirische Studien zu den Mobilitätsstrategien der Afghan/inn/en seit Beginn der kriegerischen Auseinandersetzungen belegen, dass es schwierig ist eine klare Trennlinie zwischen Flucht- und Arbeitsmigration zu ziehen (vgl. GrawerT und Mielke 2018; Monsutti 2008, 2010; Schetter 2012).

Vor dem Hintergrund der vielfältigen Migrationsformen und Mobilitätsstrategien der Afghan/inn/en scheint es wenig sinnvoll an der in Politik und Wissenschaft häufig nach wie vor vorgenommenen Differenzierung zwischen Flucht und Migration bzw. „forced“ und „voluntary migration“ festzuhalten. Viel fruchtbarer wäre es von den „Livelihood-Möglichkeiten“ betroffener Personengruppen auszugehen (vgl. GRAwERT und Mielke 2018) und Mobilität als eine mögliche Überlebensstrategie in Krisensituationen (vgl. MonsutTi 2008) zu betrachten und entsprechend zu untersuchen.

46 Eigene Beobachtungen 1991 und 1992 während Feldaufenthalten in Pakistan. Auch GRAWERT Und MIELKE (2018) nennen mehrere Beispiele dieser Arbeitsmigration von Flüchtlingen in Iran und Pakistan. 


\section{Bibliographie}

Abbasi Kh. (2015), Young Afghanistani Refugees in Iran: Professional Training, Work and Perspectives. In: Centlivres-Demont, M. (ed.), Afghanistan: Identity, Society and Politics since 1980. London, New York, I.B. Tauris, pp 275-278.

Abbasi-Shavazi M.J. and Glazebrook D. (2006), Continued Protection, Sustainable Reintegration: Afghan Refugees and Migrants in Iran. Afghanistan Research and Evaluation Unit, Kabul. $<$ https://areu.org.af/wp-content/uploads/2015/12/614E-Continued-Protection-BP-web. pdf $>$ [aufgerufen am 29.3.2019]

Abbasi-Shavazi M., Glazebrook D., Jamshidiha G., Mahmoudian H., Sadeghi R. (2008), Second-generation Afghans in Iran: integration, identity and return. Afghanistan Research and Evaluation Unit, Kabul.

$<$ https://areu.org.af/wpcontent/uploads/2016/01/823E-Second-Generation-Afghans-inIran-CS-2008.pdf $>$ [aufgerufen am 18.12.2017]

Adelkahah F. and Olszewska Z. (2007), The Iranian Afghans, Iranian Studies 40 (2), pp. 137 165.

Afghanistan Analyst Network (2016), We Knew That They Had No Future in Kabul. FriedrichEbert-Stiftung, 27. April 2016.

$<$ https://www.afghanistan-analysts.org/publication/other-publications/we-knew-that-theyhad-no-future-in-kabul-why-and-how-afghan-families-decide-to-leave/ $>$ [aufgerufen am 2.4.2019]

Ahmad A. (2019), Refugees Return to Poverty, Unemployment and Despair. Afghanistan's labor market and the status of women. VIDC (Vienna Institute for International Dialogue and Cooperation) November 2018.

$<$ http://www.vidc.org//fileadmin/Bibliothek/DP/Foto_Veranst/Fanizadeh/Afghanistan 5.11.18/Afghanistan_s_labor_market_and the_status_of_women.pdf> [aufgerufen am 29.2.2019]

Ahmad A. and Fanizadeh M. (eds.) (2018), Documentation: Afghanistan in Pakistan, Diplomatic Academy of Vienna, 6 March 2018.

$<$ http://www.vidc.org/fileadmin/Bibliothek/DP/Foto_Veranst/Fanizadeh/Afgha nistan_6.3.18/VIDC_Documentation_Afghanistan_in_Pakistan_6_March_2018.pdf> [aufgerufen am 29.3.2019]

Aнmadi Sh. (2015), Exil im Iran. Afghanische Flüchtlinge sind nur als billige Arbeitskräfte willkommen. In: Hansen S. (red.), Die große Unruhe. Afghanistan und seine Nachbarn. Editon Le Monde diplomatique, Nr. 17, Berlin; pp. 74-75 und 77.

Ajam Media Collective (2015), Between Uncertainty and Paradox: Afghan Refugees in Turkey, 10. August 2015.

$<$ http://ajammc.com/2015/08/10/afghan-refugees-in-Turkey> [aufgerufen am 21.12.2015].

Alam, H.-N. (2008), Afghanische MigrantInnen in Österreich: empirische Untersuchung zu Integration, Repatriierung und der Partizipation am Wiederaufbau des Heimatlandes. Wien, Univ., Dipl.-Arb.

AleiniKoff A.T. (2017), Toward a Global System of Human Mobility: Three Thoughts, American Journal of International Law, April 2017, pp. 24-28. < doi:10.1017/aju.2017.8>

Aurma, S. (2018), Afghan refugees in Pakistan: Harassment and deportation from women's perspectives, Spotlight 44/2018 (VIDC = Vienna Institute for International Dialogue and Cooperation). 
$<$ http://www.vidc.org/news/spotlight-442018/afghan-refugees-in-pakistan-harassmentand-deportation-from-womens-perspectives/> [aufgerufen am 29.3.2019]

Amnesty International (2017), Forced Back to Danger. Asylum-Seekers Returned from Europe to Afghanistan.

<https://www.amnesty.org/download/Documents/ASA1168662017ENGLISH.PDF> [aufgerufen am 5.3.2019]

ArJomand N. (2016), Afghan Exodus: Smuggling networks, migration and settlement patterns in Turkey. Afghanistan Analyst Network, 10 September 2016.

$<$ https://www.afghanistan-analysts.org/afghan-exodus-smuggling-networks-migrationand-settlement-patterns-in-turkey/> [aufgerufen am 30.9.2016]

Asylos (2017), Afghanistan: situation of young male "Westernised" returnees to Kabul. Asylos, August 2017.

$<$ https://asylos.eu/wp-content/uploads/2017/08/AFG2017-05-Afghanistan-Situation-ofyoung-male-Westernised-returnees-to-Kabul-1.pdf $>$ [aufgerufen am 31.3.2019]

Bakewell O. (2011), Conceptualising Displacement and Migration: Processes, Conditions, and Categories. In: Koser, Kh. and Martin, S. (eds.), The Migration-Displacement Nexus: Patterns, Processes, and Policies. New York, Oxford, Berghahn Books, pp. 14-28.

Beller M. (2013), Sicherheit im Kontext von Flucht und Asyl: Erfahrungen afghanischer Asylsuchender. Wien, Univ., Dipl.-Arb.

Berg HarpViken K. (2009), Social Networks and Migration in Wartime Afghanistan. Houndmills, Basingstoke, New York.

Berg Harpviken K. (2014), Split Return: Transnational Household Strategies in Afghan Repatriation, International Migration, 52 (6), pp. 57-71. $<$ http://onlinelibrary.wiley.com/doi/10.1111/imig.12155/epdf $>$ [aufgerufen am 14.6.2018]

BetTs A. (2013a), Survival Migration: Failed Governance and the Crisis of Displacement. Ithaca, Cornell University Press.

BetTs A. (2013b), State fragility, refugee status and 'survival migration'. In: FMR Nr. 43, May 2013, pp. 4-6.

Bezhan F. and Parsa N. (2018), Afghanistan: Things Are So Bad In Iran That Afghan Migrants Are Going Home. Radio Free Europe, Radio Liberty: August 01, 2018 16:26 GMT.

$<$ https://www.rferl.org/a/iran-s-economic-crisis-fuels-mass-exodus-of-afghanmigrants/29405068.html> [aufgerufen am 12.12.2018]

BJelica J. (2016a), Deciding to Leave Afghanistan (2): The routes and the Risks. Afghanistan Analyst Network, 19 May 2016.

$<$ https://www.afghanistan-analysts.org/wp-admin/post.php> [aufgerufen am 29.3.2019]

BJElica J. (2016b), Caught Up in Regional Tensions? The mass return of Afghan refugees from Pakistan. Afghanistan Analyst Network, 22 December 2016.

$<$ https://www.afghanistan-analysts.org/wp-admin/post.php> [aufgerufen am 29.3.2019]

BJelicA J. (2016c), Over Half a Million Afghans Flee Conflict in 2016: A look at the IDP statistics. Afghanistan Analyst Network, 28 December 2016.

$<$ https://www.afghanistan-analysts.org/over-half-a-million-afghans-flee-conflict-in-2016a-look-at-the-idp-statistics/> [aufgerufen am 29.3.2019]

BJelicA J. (2017), Voluntary and Forced Returns to Afghanistan in 2016/17: Trends, statistics and experiences. Afghanistan Analyst Network, 19 May 2017.

$<$ https://www.afghanistan-analysts.org/wp-admin/post.php> [aufgerufen am 29.3.2019] 
BJelica J. (2018), Still Caught in Regional Tensions? The uncertain destiny of Afghan refugees in Pakistan. Afghanistan Analyst Network, 31 January 2018.

$<$ https://www.afghanistan-analysts.org/wp-admin/post.php> [aufgerufen am 29.3.2019]

BMI - Sektion III Recht (Hg.) (2015), Vorläufige Asylstatistik. Vienna, Dezember 2015. $<$ http://www.bmi.gv.at/cms/BMI_Asylwesen/statistik/files/Asylstatistik_Dezember_2015.pdf>

BMI - Sektion III Recht (Hg.) (2016), Vorläufige Asylstatistik. Vienna, Dezember 2016. <http://www.bmi.gv.at/cms/BMI_Asylwesen/statistik/files/2016/Asylstatistik_ Dezember_2016.pdf $>$

BMI - Sektion III Recht (Hg.) (2017), Vorläufige Asylstatistik. Vienna, März 2017. <http://www.bmi.gv.at/cms/BMI_Asylwesen/statistik/files/2017/Asylstatistik_ Maerz_2017.pdf>

BMI - Sektion III Recht (Hg.) (2018), Vorläufige Asylstatistik, Wien Dezember 2018. $<$ https:/www.bmi.gv.at/301/Statistiken/files/2018/Asylstatistik_Dezember_2018.pdf>

Braakman M. (2005), Roots and Routes. Questions of Home, Belonging and Return in an Afghan Diaspora. M.A. Thesis, Leiden University, Department of Cultural Anthropology and Sociology of Non-Western Societies.

Brücker H., Fendel T., Kunert A., Mangold U., Siegert M., Schupp J. (2016), Geflüchtete Menschen in Deutschland. Warum sie kommen, was sie mitbringen und welche Erfahrungen sie machen. IAB-Kurzbericht 15. Nürnberg, Bertelsmann.

Castles St. (2013), The Forces Driving Global Migration, Journal of Intercultural Studies 34 (2), pp. 22-140.

Castles St., Haas de H. and Miller. M.J. (eds.) (2014), The Age of Migration: international population movements in the modern world. Basingstoke [u.a.]: Palgrave MacMillan ( $5^{\text {th }}$ edition).

Centlivres P. et Centlivres-Demont M. (1988), Et si on parlait de l'Afghanistan? Terrains et textes 1964-1980. Neuchâtel et Paris; Editions de 1'Institut d'ethnologie und Editions de la Maison des sciences de l'homme.

Centlivres P. et Centlivres-Demont M. (2000), Exil et diaspora afghane en Suisse et en Europe, CEMOTI: Cahiers d'études sur la Méditerranée orientale et le monde turco-iranien, 30, pp. 151-171.

Chatty D. and Mafleet Ph. (2013), Conceptual Problems in Forced Migration, Refugee Survey Quarterly 32 ( 2), pp. 1-13.

CHesLow D. (2016), Life in Limbo for Afghan Migrants in Turkey. RFE/RL April 24, 2016. <https:// www.rferl.org/a/afghan-migrants-turkey-life-in-limbo/27693708.html> [aufgerufen am 10.12.2016]

Clark K. (2019), Record Numbers of Civilian Casualties Overall, from Suicide Attacks and Air Strikes: UNAMA reports on the conflict in 2018. AAN, Published: 24 February 2019. $<$ https://www.afghanistan-analysts.org/wp-admin/post.php?post=25629\&action=edit \&meta-boxloader $=1 \&$ _wpnonce $=7 \mathrm{ecfa} 80954 \&$ locale $=$ user $>$ [aufgerufen am 29.3.2019]

Crawley H., Duvell F., Sigona N., McMahon S., Jones K. (2016), Unpacking a rapidly changing scenario: migration flows, routes and trajectories across the Mediterranean, MEDMIG Research Brief Nr.1, March 2016.

$<$ http://www.medmig.info/wp-content/uploads/2016/03/MEDMIG-Briefing-01-March2016-FINAL-1.pdf $>$ [aufgerufen am 12.12.2016]

CSO (Central Statistical Organization) Islamic Republic of Afghanistan (o.J.), Afghanistan Statistical Yearbook 2011-2012. < <http://cso.gov.af/en/page/1500/4722/7108> 
DaXnER M. und Nicola S.-L. (2017), Mapping of and Report on the Afghan Diaspora in Germany, Centre for International Migration and Development.

$<$ https://www.cimonline.de/static/media/cim2018-en-diaspora-afghanistan.pdf $>$ [aufgerufen am 2.11.2018]

Diop P.M. (2014), The "Bogus" Refugee: Roma Asylum Claimants and Discourses of Fraud in Canada's Bill C-31, Refuge, Canada's Journal on Refugees, 30 (1), pp. 67-80.

Donini A., Monsutti A., Scalettaris G. (2016), Afghans on the Move: Seeking Protection and Refuge in Europe. "In this journey I died several times; In Afghanistan you only die once", Global Migration Research Paper No. 17, 2016, Global Migration Centre, The Graduate Institute Geneva.

EASO (European Asylum Support Office) (2018a), EASO Country of Origin Information Report. Afghanistan Networks. no place of publication [La Valletta]; January 2018. $<$ https://coi.easo.europa.eu/administration/easo/PLib/Afghanistan_Networks.pdf > [aufgerufen am 3.2.2018]

EASO (European Asylum Support Office) (2018b), EASO Country of Origin Information Report. Afghanistan Security Situation. No place of publication [La Valletta]; May 2018. $<$ https://coi.easo.europa.eu/administration/easo/PLib/Afghanistan-security_situation _2018.pdf $>$ [aufgerufen am 3.8.2018]

Echavez Ch.R., Bagaporo J.L.L., Pilongo L.W., Azadmanesh Sh. (2014), Why do children undertake the unaccompanied journey? Motivations for departure to Europe and other industrialised countries from the perspective of children, families and residents of sending communities in Afghanistan. Afghanistan Research and Evaluation Unit and United Nations High Commissioner for Refugees, Issues Paper. Dec. 2014.

ECOI.NET (European Country of Origin Information Network) (2018), General Security Situation in Afghanistan and Events in Kabul. Published: 20 December 2018.

$<$ https:/www.ecoi.net/en/countries/afghanistan/featured-topics/general-security-situationin-afghanistan-and-events-in-kabul/> [aufgerufen am 29.3.2019]

ECRE (European Council of Refugees and Exiles) (2018), Return continues to Afghanistan in crisis. 1st June 2018.

$<$ https://www.ecre.org/return-continues-to-afghanistan-in-crisis/> [aufgerufen am 29.3.1019]

ECRE (European Council on Refugees And Exiles) (2019), No Reason for Returns to Afghanistan. ECRE's Recommendation for a Suspension of Returns to Afghanistan due to the security situation there and the unfairness of asylum decision-making in Europe. Policy Note 17, 2019.

$<$ https://www.ecre.org/ecre-policy-note-no-reason-for-returns-to-afghanistan/> [aufgerufen am 29.3.2019]

European Union External Action Service (2016), Joint Press Release: The EU and Afghanistan hold a Senior Officials' Dialogue on Migration. Bruxelles, 04/10/2016 - 20:19, UNIQUE ID: 161004_11.

$<$ https://eeas.europa.eu/headquarters/headquarters-homepage/11108/the-eu-and-afgha nistan-hold-a-senior-officials-dialogue-on-migration_en> [aufgerufen am 30.3.2019]

EUROSTAT Datenset (2018), Asylbewerber: mutmaßlich unbegleitete Minderjährige nach Staatsangehörigkeit, Alter und Geschlecht Jährliche Daten (gerundet) [migr_asyunaa]; Letzte Aktualisierung: 16-05-2018.

$<$ http://appsso.eurostat.ec.europa.eu/nui/show.do?dataset $=$ migr_asyunaa\&lang=de $>$ [aufgerufen am 30.5.2018] 
EUROSTAT Newsrelease (2017), Asylum applicants considered to be unaccompanied minors. 63 300 unaccompanied minors among asylum seekers registered in the EU in 2016. Over half are Afghans or Syrians. Eurostat Newsrelease 80/2017; 11 May 2017.

$<$ http://ec.europa.eu/eurostat/documents/2995521/8016696/3-11052017-AP-EN. pdf/30ca2206-0db9-4076-a681-e069a4bc5290>,

$<$ http://www.europeanmigrationlaw.eu/documents/Asylum\%20applicants $\% 20$ considered $\% 20$ to $\% 20 \mathrm{be} \% 20$ unaccompanied $\% 20$ minors.pdf $>$ [aufgerufen am 30.5.2018]

EUROSTAT-Pressemitteilung (2016), Asyl in den EU-Mitgliedstaaten: Rekordzahl von über 1,2 Millionen registrierten erstmaligen Asylbewerbern im Jahr 2015. Syrer, Afghanen und Iraker an erster Stelle. Eurostat-Pressemitteilung 44/2016; 4. März 2016.

$<$ http://ec.europa.eu/eurostat/documents/2995521/7203837/3-04032016-AP-DE. PDF/9FCD72AD-C249-4F85-8C6D-E9FC2614AF1B > [aufgerufen am 12.7.2018]

EUROSTAT-Pressemitteilung (2017), Asyl in den EU-Mitgliedstaaten: 1,2 Millionen erstmalige Asylwerber im Jahr 2015 registriert. Syrer, Afghanen und Iraker weiterhin an erster Stelle. Eurostat-Pressemitteilung 46/2017; 16. März 2017.

$<$ http://ec.europa.eu/eurostat/documents/2995521/7921614/3-16032017-BP-DE. pdf/28884e8a-bb55-48bf-85b6-b4157f131c55> [aufgerufen am 12.7.2918]

EUROSTAT-Pressemitteilung (2018), Asyl in den EU-Mitgliedstaaten. 650000 erstmalige Asylbewerber im Jahr 2017 registriert Syrer, Iraker und Afghanen weiterhin an erster Stelle. Eurostat-Pressemitteilung 47/2018: 20.März 2018.

$<$ http://ec.europa.eu/eurostat/documents/2995521/8754393/3-20032018-AP-DE. pdf/72fe7d90-d966-425a-832f-28dc3a4cd2e6> [aufgerufen am 12.7.2018]

EUROSTAT-Pressemitteilung (2019), Asyl in den EU-Mitgliedstaaten. 580800 erstmalige Asylbewerber im Jahr 2018 registriert, Rückgang um 11\% gegenüber 2017. Syrer, Afghanen und Iraker weiterhin an erster Stelle. Eurostat- Pressemitteilung 46/2019: 14.März 2019. $<$ https://ec.europa.eu/eurostat/documents/2995521/9665551/3-14032019-AP-DE.pdf/ c10346dd-55b9-4bb0-aacb-f13be848efaa > [aufgerufen am 27.3.2019]

EUROSTAT Statistics explained (2019a), Statistics on enforcement of immigration legislation. Statistics Explained, Data extracted in June 2018. 6. März 2019.

$<$ https://ec.europa.eu/eurostat/statistics-explained/pdfscache/37449.pdf $>$ [aufgerufen am 27.3.2019]

EUROSTAT Statistics explained (2019b), Main trends in the numbers of asylum applicants. Asylum quarterly report, Statistics Explained, Data extracted on 12 March 2019. 22. März 2019

$<$ https://ec.europa.eu/eurostat/statistics-explained/pdfscache/13562.pdf $>$ [aufgerufen am 27.3.2019]

Fiddian-Qasmiyeh E., Loescher G., Long K. and Sigona N. (2014), Introduction: Refugee and Forced Migration Studies in Transition. In: Fiddian-QAsmiYeh E., Loescher G., Long K. and Sigona N. (eds.), The Oxford Handbook of Refugee and Forced Migration Studies. Oxford etc., Oxford University Press; pp. 1-14.

FMR (Forced Migration Review) (2014), Afghanistan's displaced people: 2014 and beyond, FMR 46, May 2014

$<$ http://www.fmreview.org/sites/fmr/files/FMRdownloads/en/afghanistan.pdf $>$ [aufgerufen am 29.3.2019]

Foschini F. (2017), Afghan Child Migrants: Italy, the preferred country of transit? Afghanistan Analyst Network, 6 September 2017.

$<$ https://www.afghanistan-analysts.org/afghan-child-migrants-italy-the-preferred-countryof-transit/?format=pdf $>$ [aufgerufen am 23.3.2019] 
Friedrich D., Kohlbacher J., Lehner M., Rasuly-Paleczek G., Röhm M. (2019), Endbericht: Integrationsverläufe Asylberechtiger und subsidiär Schutzberechtiger aus Afghanistan in Österreich: Veränderungsprozesse der Wertvorstellungen und Erwartungshaltungen. Wien [31.7.2018]

Gehrig T. (1999), La diaspora afghane au Pakistan et en Europe : réseaux et stratégies des élites urbaines, Tsantsa, Zeitschrift der Schweizerischen Ethnologischen Gesellschaft = Revue de la Société suisse d'ethnologie, 4 (183).

Giles W. (2010), Livelihood and Afghan refugee workers in Iran. In: Lem W. and Gardiner BARBER P. (eds.), Class, contention, and a world in motion. New York, Berghahn Books, pp. 23-40.

Glatz, A.-K. (2015), Afghanistan: New and long-term IDP's risk becoming neglected as conflict intensifies. (IDMC = Internally Displaced Monitoring Centre, 16th. July 2015).

<http://www.internal-displacement.org/south-and-south-east-asia/afghanistan/2015/ afghanistan-new-and-long-term-idps-risk-becoming-neglected-as-conflict-intensifies> [aufgerufen am 29.3.2019]

GraWERT E. (2018), Rückkehr afghanischer Flüchtlinge aus Iran. Bpb Bundeszentrale für politische Bildung. 16.10.2018.

$<$ http://www.bpb.de/gesellschaft/migration/laenderprofile/277617/rueckkehr-afghani scher-fluechtlinge $>$ [aufgerufen am 9.3.2019]

Grawert E. and Mielke K. (2018a), Coping with protracted displacement. How Afghans secure their livelihood in Afghanistan, Iran and Pakistan. BICC-Working Paper Nr. 2/2018; Bonn, BICC (Bonn International Center for Conversion).

GrötZBACH E. (1972), Kulturgeographischer Wandel in Nordost-Afghanistan seit dem 19. Jahrhundert. Meisenheim am Glan, Verlag Anton Hain.

GrötzвACH E. (1990), Afghanistan: eine geographische Landeskunde. Darmstadt: Wissenschaftliche Buchgesellschaft.

HАкімі H. (2016), Understanding the Drivers of Migration to Europe: Lessons from Afghanistan for the Current Refugee Crisis. London, The Royal Institute of International Affairs.

Hofmann M. und Reichel D. (2014), Migration from Afghanistan to Europe: Statistical Overview. In: Taucher W., Vogl M., Webinger P. (eds.), Afghanistan: 2014 and Beyond. Wien, Austrian Federal Ministry of the Interior; pp. 102-116.

Hoodfar H. (2010), Refusing the Margins: Afghan Refugee Youth in Iran. In: Chatty, D. (ed.): Deterritorialized youth: Sahrawi and Afghan refugees at the margins of the Middle East. New York [u.a.], Berghahn Books, pp.145-181.

Houte M. van (2016), Return Migration to Afghanistan. Moving Back or Moving Forward? Cham, Palgrave Macmillan.

Houte, M. van, Siegel M., Davids T. (2015), Return to Afghanistan: Migration as Reinforcement of Socio-Economic Stratification, Population, Space and Place, Vol. 21, pp. 692-703.

Human Rights Watch (2013), Unwelcome Guests. Iran's Violation of Afghan Refugee and Migrant Rights. Nov. 2013.

$<$ https://www.hrw.org/sites/default/files/reports/iran1113_forUpload_0.pdf> [aufgerufen am 29.3.2019]

Human Rights Watch (2017), Pakistan coercion, UN complicity. The Mass Forced Return of Afghan Refugees. February 2017.

$<$ https://www.hrw.org/sites/default/files/report_pdf/pakistan0217_web.pdf> [aufgerufen am 29.3.2019] 
IBrahimi N. (2017), The Hazaras and the Afghan State. Rebellion, Exclusion and the Struggle for Recognition. London, Hurst.

IDMC (International Displacement Monitoring Centre) (2017), GRID Global Report on Internal Displacement. Geneva, May 2017

$<$ http://www.internal-displacement.org/global-report/grid2017/> [aufgerufen am 29.3.2019]

iDMC (International Displacement Monitoring Centre) (2018), Afghanistan. $<\mathrm{http}$ ://www.internal-displacement.org/countries/Afghanistan $>$ [aufgerufen am 2.11.2018]

IOM (International Organization for Migration) (2016), Glossary on Migration. Geneva, $3^{\text {rd }}$ edition

IOM (International Organization for Migration) (2019a), Afghanistan. A country on the Move. Geneva.

$<$ https://publications.iom.int/books/afghanistan-country-move> [aufgerufen am 2.2.2019]

IOM (International Organization for Migration) (2019b), DTM Afghanistan. Baseline Mobility Assessment, Summary Results Round 7, Oct -Dec 2018. [published mid-March 2019] $<$ https://displacement.iom.int/system/tdf/reports/IOM-Afghanistan-Baseline-MobilityAssessment-Summary-Results-December-2018-English.pdf?file=1\&type=node\&id=5322> [aufgerufen am 21.3.2019]

IOM and UNHCR (2018), Returns to Afghanistan in 2017. Joint IOM-UNHCR Summary Report. Kabul, 28. February 2018.

$<$ https://afghanistan.iom.int/sites/default/files/Reports/joint_returnee_report_iom_unhcr_ final.pdf $>$ [aufgerufen am 21.3.2019]

Kabir M. (2015), Diskriminierung im Alltag. In: Hansen, S. (red.): Die große Unruhe. Afghanistan und seine Nachbarn. Editon Le Monde diplomatique, Nr. 17, Berlin, pp. 76-77.

Kayadglu T. (2015), Silent crisis'for Turkey's Afghan refugees: Afghans claim their situation has worsened as international community struggles to cope with millions fleeing Syrian war. 8. Juli 2015. [Anadolu Agency]

$<\mathrm{http}$ //aa.com.tr/en/life/silent-crisis-for-turkeys-afghan-refugees/28237> [aufgerufen am 29.3.2019].

Kaytaz E.S. (2016), Afghan Journeys to Turkey: Narratives of Immobility, Travel, and Transformation, Geopolitics, 21 (2), pp. 284-302.

Kazemi S.R. (2016), Afghan Exodus: Maruf's tale of an emerging transnational community between Herat and Europe. Afghanistan Analyst Network, 17 September 2016. $<$ https://www.afghanistan-analysts.org/afghan-exodus-marufs-tale-of-an-emerging-trans national-community-betweenherat-and-europe/?format $=$ pdf $>$ [aufgerufen am 29.3.2019].

Kepp C., Nakschbandi D., Riebau M., Schmidt V. (red.) (2018), Rückkehr ins Ungewisse. Von Europa nach Afghanistan: Erfahrungsberichte von Kindern und Jugendlichen. Save the Children Deutschland e.V. (ed.), Okt. 2018.

KLeIST O.J. (2015a), Über Flucht forschen. Herausforderungen der Flüchtlingsforschung, Peripherie, Nr. 138/139; 35. Jahrgang; pp. 150-169.

KLEIST O.J. (2015b), Warum weit weniger Asylbewerber in Europa sind, als angenommen wird: Probleme mit den Eurostats Asylzahlen, ZAR 9, pp. 294-299.

KLeist O.J. (2018a), Mixed Migration: Der Flüchtling als politisch und ethische Figur, Ethik und Unterricht, 2, pp. 10-13.

KLeist O.J. (2018b), “The Refugee Regime: Sovereignty, Belonging and the Political of Forced Migration". In: Pотт A., Rass Ch. und WolfF F. (eds.), Was ist ein Migrationsregime? What Is a Migration Regime? Wiesbaden, Springer V.S., pp. 167-185. 
Kohlbacher J. und Rasuly-Paleczek G. (2018), From Destination to Integration - First Experiences of Asylum Seekers from Austria, Syria, and Iraq arriving in Austria. In: CARVILLSchellenbacher J., Dahlvik, J., Fassmann H., und Reinprecht Ch., (eds.), Migration und Integration - wissenschaftliche Perspektiven aus Österreich. Jahrbuch 4/2018. Wien, Vienna University Press, pp. 31-49.

Koser Kh. and Martin S. (2011), The Migration-Displacement Nexus. In: Koser Kh. and Martin S. (eds.), The Migration-Displacement Nexus. Patterns, Processes, and Policies. New York, Oxford, Berghahn Books, pp. 1-13.

Kronenfeld D.A. (2008), Afghan Refugees in Pakistan: Nor All Refugees, Not Always in Pakistan, Not Necessarily Afghan?. Journal of Refugee Studies, 21 (1), pp. 43-63.

LinKe L. (2016), Deciding to Leave Afghanistan (1): Motives for migration. Afghanistan Analyst Network; Published: 8 May 2016. $<$ https://www.afghanistan-analysts.org/wp-admin/post.php> [aufgerufen am 29.3.2019]

MaJidi N. (2018), Deportees Lost at "Home": Post-deportation Outcomes in Afghanistan. In: Khosravi Sh. (ed.), After Deportation. Ethnographic Perspectives. Cham, Palgrave Macmillan, pp. 127-148.

Majidi N., Van der Vorst V. and FoukLes Ch. (2016), Seeking Safety, Jobs, and More: Afghanistan's Mixed Flows Test Migration Policies, February 25, 2016.

$<$ https://www.migrationpolicy.org/article/seeking-safety-jobs-and-more-afghanistan $\%$ E2\%80\%99s-mixed-flows-test-migration-policies> [aufgerufen am 29.3.2019]

Marsden M. (2016), Trading Worlds: Afghan Merchants across Modern Frontiers. Hurst and Company, London, UK.

MatzenBerger M. und Minkin Ch. (2016), Wirtschaftsflüchtlinge: Eine Kategorie ohne Definition, Der Standard, 14. Jänner 2016.

$<$ https://derstandard.at/2000029008447/Wirtschaftsfluechtlinge-Eine-Kategorie-ohneDefinition> [aufgerufen am 29.3.2019]

Migration Data Portal (2019a), Stichwort Forced Migration and Displacement. [last updated 20.Febr.2019].

$<$ https://migrationdataportal.org/themes/forced-migration-or-displacement\#definitions $>$ [aufgerufen am 6.3.2019]

Migration Data Portal (2019b), Stichwort: Environmental Migration. [last updated 8. Febr. 2019].

$<$ https://migrationdataportal.org/themes/environmental_migration $>$ [aufgerufen am 6.3.2019]

Mixed Migration Centre (2019), Distant Dreams. Understanding the aspirations of Afghan Returnees. January 2019, Published 12 March 2019.

$<$ http://www.mixedmigration.org/wp-content/uploads/2019/02/061_Distant_Dreams.pdf $>$ [aufgerufen am 21.3.2019]

Monsutti A. (2005), War and Migration: Social Networks and Economic Strategies of the Hazaras of Afghanistan. New York \& London, Routledge.

Monsutti, A. (2008), Afghan Migratory Strategies and the Three Solutions to the Refugee Problem. Refugee Survey Quarterly, 27 (1), pp. 58-73.

Monsutti A. (2010), The Transnational Turn in Migration Studies and the Afghan Social Networks. In: Chatty D. and Finlayson B. (eds.), Dispossession and Displacement. Forced Migration in the Middle East and North Africa. Cambridge etc., Cambridge Univ. Press, pp. 45-67. 
Monsuttr A. (2018a), Mobility as a political act. Ethnic and Racial Studies, 41 (3); pp. 448-455.

Monsutti A. (2018b), Homo itinerans. La planète des Afghans. Paris, Presses Universitaires de France.

NABy E. (2005), The Afghan Diaspora: Reflection on the Imaged Country. In: AtABAKI T. and Mehendale S. (eds.), Central Asia and the Caucasus. Transnationalism and Diaspora. London, New York, Routledge, pp. 169-183.

Naseh M., Potocky M., Stuart P.H. and Pezeshk S. (2018), Repatriation of Afghan Refugees from Iran: a shelter profile study. Journal of International Humanitarian Action, 3 (13), pp. 1-12.

Neumayer E. (2005), Bogus Refugees? The Determinants of Asylum Migration to Western Europe. International Studies Quarterly, 49 (3), pp. 389-409. $<$ https://www.jstor.org/stable/3693600>

Nicolini A. (2013), Safar ba London. Afghan youths on the move. Afghanistan Analyst Network, 6. August 2014.

$<$ http://www.afghanistan-analysts.org/safar-ba-lonon-afghan-youths-on-the-move> [aufgerufen am 11.12.2013]

OCHA (United Nations Office for the Coordination of Humanitarian Affairs) (2015), Afghanistan. Human Needs Overview 2016 (published in Nov. 2015).

$<$ https://www.humanitarianresponse.info/system/files/documents/files/afg_2016_hno_ final_20151209.pdf $>$ [aufgerufen am 15.5.2017]

OCHA (UN Office for the Coordination of Humanitarian Assistance) (2017a), Afghanistan: Humanitarian Needs Overview 2018, Dec. 2017.

$<$ https://reliefweb.int/sites/reliefweb.int/files/resources/afg_2018_humanitarian_needs_ overview_1.pdf $>$ [aufgerufen 24.07.2018]

OCHA (UN Office for the Coordination of Humanitarian Affairs) (2017b), Pakistan: Afghan Refugees and Undocumented Afghans Repatriation (21-27 May 2017).

$<$ https://www.humanitarianresponse.info/system/files/documents/files/afghan returns_20170523_0.pdf> [aufgerufen am 24.07.2018]

OCHA (UN Office for the Coordination of Humanitarian Affairs) (2018a), Afghanistan: Conflict Induced Displacements (as of 31 October 2018).

$<$ https://www.humanitarianresponse.info/en/operations/afghanistan/idps $>$ [aufgerufen am 2.11.2018]

OCHA (UN Office for the Coordination of Humanitarian Affairs) (2018b), In Afghanistan Weekly Field Report 24. Dec. to 31 Dec 2018.

$<$ https://reliefweb.int/report/afghanistan/afghanistan-weekly-field-report-24-december-31december-2018> [aufgerufen am 27.3.2019]

OCHA (UN Office for the Coordination of Humanitarian Affairs) (2018c), Afghanistan: Overview of Natural Disasters (as of 22 October). Natural Disaster Events from 2 January 2018 to 8 October 2018.

$<$ https://www.humanitarianresponse.info/en/operations/afghanistan/natural-disasters-0> [aufgerufen am 24.10.2018]

OCHA (UN Office for the Coordination of Humanitarian Affairs) (2019), Afghanistan: Conflict Induced Displacements (as of 6 January 2019).

$<$ https://www.humanitarianresponse.info/en/operations/afghanistan/idps $>$ [aufgerufen am 9.1.2019]

Orywal E. (1986), Die ethnischen Gruppen Afghanistans. Fallstudien zu Gruppenidentität und Intergruppenbeziehungen. Wiesbaden, Reichert. 
Ozlatimoghaddam M. (2012), "We Live Like Sea Foam": Experiences of Liminality Among Afghan Migrants in Ceza City-Turkey. MA thesis, The Graduate School of Social Sciences, METU, Ankara.

Parusel B. (2018), Afghanische Asylsuchende und das Gemeinsame Europäische Asylsystem. bpb (Bundeszentrale für politische Bildung) 16.10.2018.

$<$ http://www.bpb.de/gesellschaft/migration/laenderprofile/277690/das-> [aufgerufen am 23.3.2019]

Picozza F. (2017), Dubliners: Unthinking Displacement, Illegality, and Refugeeness within Europe's Geographies of Asylum. In: De Genova N. (ed.), The Borders of "Europe". Autonomy of Migration, Tactics of Bordering. Durham and London, Duke University Press, pp. 233-254.

Rasuly S. (1997), Die politischen Eliten Afghanistans. Ihre Entstehungsgeschichte, ihre Bedeutung und ihr Versagen in der Gegenwart. Frankfurt am Main etc., Peter Lang Verlag.

Rasuly-Paleczek G. (2001), Afghanische Flüchtlinge - von der Welt vergessen, Asylkoordination Aktuell, 1, pp. 27-40.

Rasuly-Paleczek G. (2004), Frontiers, Hinterlands, Centers, Peripheries: Adapting to Changing Fortunes - the Uzbeks of Afghanistan. In: Rasuly-Paleczek G. and Kaschnig J. (eds.), Central Asia on Display. Proceedings of the VII. Conference of the European Society for Central Asian Studies. Vienna (LIT-Publishers), pp. 81-108.

Rasuly-Paleczek G. (2017), Many Reasons for Leaving Afghanistan: Social Obligations in Times of Protracted Violence. In: Kohlbacher J. and Schiocchet L. (eds.), From Destination to Integration - Afghan, Syrian and Iraqi Refugees in Vienna. ISR-Forschungsbericht 45. Wien, Verlag ÖAW, pp. 57-83.

Reichel D. und Hofmann M. (2011), Migrationsströme aus Afghanistan und Pakistan nach Europa und Österreich. In: Janda A.; Taucher W. und Vogl M. (eds.), AfPak. Afghanistan, Pakistan und die Migration nach Österreich. Wien, ÖIF und BM für Inneres, 2011, pp. 9-28.

RiesenkampfF M. and Noor Ebad A. (2017), Die aktuelle Migration aus Afghanistan: Hintergründe und Ursachen. Länderberichte Konrad-Adenauer-Stiftung e.V. Februar 2017. $<\mathrm{http} / /$ www.kas.de/afghanistan/de/publications/48261/> [aufgerufen am 23.3.2019]

Rostami-Povey E. (2007), Afghan Refugees in Iran, Pakistan, the U.K. and the U.S. and Life after Return: A Comparative Gender Analysis, Iranian Studies, Vol. 40, No. 2, pp. 241-261. $<$ DOI: $10.1080 / 00210860701269576>$

RutTIG Th. (2017a), Afghan Exodus: Afghan asylum seekers in Europe (1) - the changing situation. Afghanistan Analyst Network, 13. February 2017.

$<$ https://www.afghanistan-analysts.org/afghan-exodus-afghan-asylum-seekers-in-europe1-the-changingsituation/?format=pdf $>$ [aufgerufen am 29.5.2018]

RutTig Th. (2017b), Afghan Exodus: Afghan asylum seekers in Europe (2) - the north-south divide. Afghanistan Analyse Network, 15 February 2017.

$<$ https://www.afghanistan-analysts.org/afghan-exodus-afghan-asylum-seekers-in-europe2-the-north-southdivide/? format=pdf $>$ [aufgerufen am 29.5.2018]

RutTIG Th. (2017c), Afghan Exodus: Afghan asylum seekers in Europe (3) - case study Germany. Afghanistan Analyst Network, Published: 17 February 2017.

$<$ https://www.afghanistan-analysts.org/wp-admin/post.php> [aufgerufen am 29.5.2018].

Ruttig Th. (2017d), Pressure and Peril: Afghan refugees and Europe in 2017. Afghanistan Analyst Network: Published: 30 December 2017.

$<$ https://www.afghanistan-analysts.org/wp-admin/post.php?t=1514845606819> [aufgerufen am 29.5.2018] 
RutTig Th. (2018a), More violent, more widespread: Trends in Afghan security in 2017. In: Afghanistan Analyst Network, Published: 29 January 2018.

$<$ https://www.afghanistan-analysts.org/wp-admin/post.php $>$ [aufgerufen am 11.7.2018]

Ruttig Th. (2018b), Five Questions to Make Sense of the New Peak in Urban Attacks and a Violent Weekend in Kabul. Afghanistan Analyst Network, 5.February 2018.

$<$ https://www.afghanistan-analysts.org/wp-admin/post.php> [aufgerufen am 15.7.2018]

SADat S.M. (2017), What Factors Drive Child Marriage in Afghanistan? The Asia Foundation, March 22, 2017.

$<$ https://asiafoundation.org/2017/03/22/factors-drive-child-marriage-afghanistan/> [aufgerufen am 2.4.2019]

SAFI M. (2016), Youth migration - a result of the Afghan government's failed peace policy. Post auf Website transconflict vom 21 March 2916.

$<$ http://www.transconflict.com/2016/03/youth-migration-result-afghan-governmentsfailed-peace-policy.213/> [aufgerufen am 2.4.2019]

Saito M. (2007), Second-Generation Afghans in Neighbouring Countries: From Mohajer to Hamwatan: Afghans Return Home. Kabul, AREU, 2007.

$<$ https://www.refworld.org/pdfid/47c3f3cbd.pdf> [aufgerufen am 2.4.2019]

Saito M. (2009), Searching for My Homeland: Dilemmas Between Borders. Experiences of Young Afghan Returning "Home" From Pakistan and Iran. Afghanistan Research and Evaluation Unit, Synthesis Paper Series, July 2009.

$<$ https://www.ecoi.net/en/file/local/1287640/1226 1249306039 experiences-of-youngafghans-returning-home-from-pakistan-and-iran-2009.pdf $>$ [aufgerufen am 2.4.2019]

Samuel Hall, NRC, IDMC (2018), Challenges to IDPs' Protection in Afghanistan, commissioned by NRC / IDMC and funded by the European Union and the Norwegian Ministry of Foreign Affairs.

$<$ https://www.nrc.no/globalassets/pdf/reports/escaping-war---where-to-next/nrc_idp escaping-war_where-to-next.pdf $>$ [aufgerufen am 23.3.2019]

SCHEIBELHofer P. (2017), 'It won't work without ugly pictures': images of othered masculinities and the legitimisation of restrictive refugee-politics in Austria. NORMA, International Journal for Masculinity Studies, Vol. 12, No. 2, pp. 96-111. $<$ https://doi.org/10.1080/18902138.2017.1341222>

Schetter C. (2012), Translocal Lives. Patterns of Migration in Afghanistan. Crossroads Asia, Working Paper Series No. 02, Bonn 3. Jänner 2012.

SchmeIdL S. (1997), Exploring the Causes of Forced Migration: A Pooled Time-Series 1971-1990. Social Science Quarterly, Vol. 78, No. 2, pp. 284-308.

SchmeIdL S. (2019), Vier Jahrzehnte afghanischer Flucht und Vertreibung. bpb (Bundeszentrale für politische Bildung) 10.1.2019.

$<$ http://www.bpb.de/gesellschaft/migration/laenderprofile/283689/vier-jahrzehnteafghanischer-flucht-und-vertreibung > [aufgerufen am 29.3.2019]

Seyhan S. (2017), The Evolution of Afghan Migration in Istanbul. Harekact, Indepth. $<$ http://harekact.bordermonitoring.eu/2017/12/17/the-evolution-of-afghan-migration-inistanbul/> [aufgerufen am 29.5.2018]

SigAR (Special Inspector General for Afghanistan Reconstruction Report) (2015), Afghan Refugees and Returnees: Corruption and Lack of Afghan Ministerial Capacity Have Prevented Implementation of a Long-term Refugee Strategy. August.

$<$ https://www.sigar.mil/pdf/audits/SIGAR-15-83-AR.pdf $>$ [aufgerufen am 29.5.2018] 
SigAR (Special Inspector General for Afghanistan Reconstruction) (2018), Quarterly Report to Congress, 30 October 2018.

$<$ https://www.sigar.mil/pdf/quarterlyreports/2018-10-30qr-section3-security.pdf> [aufgerufen am 29.3.2019]

SLiwinski M. (1988), Afghanistan 1978-87, War, Demography and Society. Central Asian Survey, Incidential Papers Series, No. 6.

STAHLmann F. (2017), Überleben in Afghanistan? Zur humanitären Lage von Rückkehrenden und ihren Chancen auf familiäre Unterstützung, Asylmagazin 3, pp. 73-81. $<$ https://www.nds-fluerat.org/wp-content/uploads/2017/03/AM17-3_U\%CC\%88berlebenin-Afghanistan_Stahlmann.pdf $>$ [aufgerufen am 2.4.2019].

TAPPer N. (1983), Abd-al-Rahman's North-West-Frontier. The Pashtun Colonisation of Afghan Turkestan. In: TAPPER, R. (ed.): Conflict of Tribe and State in Iran and Afghanistan. London, Canberra, New York, St. Martin's Press, pp. 233-262.

TIETJENS H. (2002), Rückkehrerprojekte für in Deutschland lebende Afghanen/-innen im Bereich der Erwachsenenbildung. Institut für internationale Zusammenarbeit des Deutschen Volkshochschulverbandes (IIZ/DVV). Institut für international und interkulturell vergleichende Erziehungswissenschaft, Arbeitsbereich Interkulturelle Bildungsforschung der Universität Hamburg.

Treichler R. und Gepp J. (2015), Wirtschaftsflüchtlinge: Die (ganz besonders) Unerwünschten, Profil vom (27.8.2015).

$<$ https://www.profil.at/ausland/wirtschaftsfluechtlinge-unerwuenschten-5830954> [aufgerufen am 23.3.2019]

Turton D. (2003a), Conceptualising Forced Migration, RSC Working Paper. Oxford.

TURTON D. (2003b), Refugees, forced resettlers and 'other forced migrants': towards a unitary study of forced migration. Genf.

Turton D. and Marsden P. (2002), Taking Refugees for a Ride? The politics of refugee return to Afghanistan. In: Issues Paper Series: AREU Afghanistan Research and Evaluation Unit.

UNAMA (United Nations Assistance Mission in Afghanistan) (2019a), Civilian deaths from Afghan conflict in 2018 at highest recorded level - UN report, 24 Feb 2019. $<$ https://unama.unmissions.org/civilian-deaths-afghan-conflict-2018-highest-recordedlevel-\%E2\%80\%93-un-report> [aufgerufen am 5.3.2019]

unama (United Nations Assistance Mission in Afghanistan and United Nation Human Rights Office of the High Commissioner) (2019b), Afghanistan: Protection of Civilians in Armed Conflict: Annual Report 2018. Kabul, Februar 2019.

$<$ https://unama.unmissions.org/sites/default/files/afghanistan_protection_of_civilians_ annual_report2018_final_24_feb_2019_1.pdf> [aufgerufen am 5.3.2019]

UNHCR (2010), Trees Only Move in the Wind A Study of Unaccompanied Afghan Children in Europe. Christine Mougne (author of report). Geneva, Policy Development and Evaluation Service.

$<$ http://www.unhcr.org/4c1229669.html> [aufgerufen am 19.09.2012]

UNHCR (2018a), Global Trends: Forced Displacement in 2017. Geneva, 20. June 2018. $<$ https://www.unhcr.org/5b27be547.pdf $>$ [aufgerufen am 5.3.2019]

UNHCR (2018b), Solutions Strategy for Afghan Refugees 2018-2019. Enhancing Resilience and Co-Existence through Greater Responsibility-Sharing. Geneva, October 2018. $<$ https://data2.unhcr.org/en/documents/download/66534> [aufgerufen am 21.3.2019] 
Verma S. (2018), Mass Deportations of Afghans from Turkey: Thousands of migrants sent back in a deportation drive. Afghanistan Analyst Network, 21 June 2018.

$<$ https://www.afghanistan-analysts.org/mass-deportations-of-afghans-from-turkey/> [aufgerufen am 13.7.2018]

Vogelsang W. (2002), The Afghans. Oxford [u.a.], Blackwell.

WaIt E. (2018), Killing Time in Bosnia and Herzegovina: Afghan migrants try a new route into the EU. Afghanistan Analyst Network, 4. Sept 2018.

$<$ https://www.afghanistan-analysts.org/killing-time-in-bosnia-and-herzegovina-afghanmigrants-try-a-new-route-into-the-eu/ $>$ [retrieved 21.3.2019]

WiLKENS A. (2016), An Afghan Exodus (2): Unaccompanied minors in Sweden. AAN Afghanistan Analysts Network, 18.02.2016.

$<$ https://www.afghanistan-analysts.org/an-afghan-exodus-2-unaccompanied-minors-insweden/> [aufgerufen am 2.4.2019]

Willner-Reid M. (2017), Afghanistan: Displacement Challenges in a Country on the Move. November 16, 2017.

$<$ https://www.migrationpolicy.org/article/afghanistan-displacement-challenges-countrymove?utm_source $=$ Monthly + News + Update $\% 2 \mathrm{C}+$ November $+2017 \&$ utm_campaign $=$ Mon thly + News + Update + November $+2017 \&$ utm_medium $=$ email $>$ [aufgerufen am 23.3.2019]

Zetter R. (2007), More Labels, Fewer Refugees: Remaking the Refugee Label in an Era of Globalization. Journal of Refugee Studies, 20 (2), pp. 172-192.

ZIECK M. (2008), The Legal Status of Afghan Refugees in Pakistan, a Story of Eight Agreements and Two Suppressed Premises. International Journal of Refugee Law, 20 (2), pp. 253-272. $<$ http://ijrl.oxfordjournals.org/cgi/content/abstract/20/2/253> 